\title{
Representational momentum and related displacements in spatial memory: A review of the findings
}

\author{
TIMOTHY L. HUBBARD \\ Texas Christian University, Fort Worth, Texas
}

\begin{abstract}
Memory for the final location of a moving target is often displaced in the direction of target motion, and this has been referred to as representational momentum. Characteristics of the target (e.g., velocity, size, direction, and identity), display (e.g., target format, retention interval, and response method), context (landmarks, expectations, and attribution of motion source), and observer (e.g., allocation of attention, eye movements, and psychopathology) that influence the direction and magnitude of displacement are reviewed. Specific conclusions regarding numerous variables that influence displacement (e.g., presence of landmarks or surrounding context), as well as broad-based conclusions regarding displacement in general (e.g., displacement does not reflect objective physical principles, may reflect aspects of naive physics, does not solely reflect eye movements, may involve some modular processing, and reflects high-level processes) are drawn. A possible computational theory of displacement is suggested in which displacement (1) helps bridge the gap between perception and action and (2) plays a critical part in localizing stimuli in the environment.
\end{abstract}

An observer's memory for the final position of a previously viewed moving target is often displaced forward in the direction of target motion. This displacement has been suggested to reflect the implied momentum of the target and has been referred to as representational momentum (Freyd \& Finke, 1984; for a sampling of recent research, see Thornton \& Hubbard, 2002). Momentum is one of several physical principles that has been suggested to influence memory for the position of a target, and evidence previously interpreted as suggesting that momentum and other physical principles influence spatial representation, as well as evidence regarding other variables that influence this displacement, will be reviewed. Although it may seem counterintuitive that such a distortion or bias in mental representation could be adaptive, such displacement may play an important role in the spatial localization of stimuli by helping to bridge the gap between perception and action. Creating such a bridge could help observers localize and optimally respond to stimuli, and so such displacement in the mental representation of the final position of a target could be a critical part of a larger computational theory of perception.

The author thanks Mary Hegarty, Scott Jordan, Mary Kaiser, Dirk Kerzel, Michael Motes, Greta Munger, Suparna Rajaram, Ian Thornton, and two anonymous reviewers for comments on a previous version of the manuscript. Correspondence may be addressed to T. L. Hubbard, Department of Psychology, Texas Christian University, Box 298920, Fort Worth, TX 76129 (e-mail: t.hubbard@tcu.edu).

\section{PART I Overview of Displacement}

In early studies of displacement, researchers used the term representational momentum to refer to any mislocalization of the remembered final position of a moving target in the direction of target motion. However, there are a number of potential issues with this term. First, and as will be discussed in more detail below, mislocalization of the final position of a moving target in the direction of motion is influenced by variables other than the implied momentum of the target. The term representational momentum does not accurately describe a combination of momentum and nonmomentum variables, nor does it distinguish between effects of implied momentum and effects of other variables. Second, the term representational momentum has been used to refer to displacement along any dimension of change, even when change along that dimension might not be associated with physical momentum (e.g., auditory frequency, Freyd, Kelly, \& DeKay, 1990; brightness, Brehaut \& Tipper, 1996; facial expression, Thornton, 1998). Third, the term representational momentum has been used to describe a forward mislocalization of the final position of a moving target and also as an explanatory mechanism for that forward mislocalization, and this has resulted in a blurring of data and theory. Fourth, mislocalization of the final position of a moving target can occur in directions other than the direction of target motion, and mislocalization in these other directions would not reflect effects of momentum. 
Given the issues associated with the term representational momentum, Hubbard (1995c) has suggested that the broader term displacement should be used to refer to general mislocalizations in memory for the final position of a target, and the more specific term representational momentum should be used only to refer to that component of displacement that is attributed to the implied momentum of the target. The momentum of a physical object is defined as the product of that object's mass and velocity (i.e., momentum $=$ mass $\times$ velocity), but neurons representing the motion of a physical object would not themselves actually be in motion (just as neurons representing a mental rotation would not themselves actually be rotating), and so mental representations would not be expected to possess physical momentum per se. In the following discussion, the term representational momentum is used to describe that component of displacement consistent with how physical momentum would influence a physical object, but such a use is necessarily more abstract and metaphorical than concrete and literal (i.e., more consistent with second-order isomorphism than with first-order isomorphism; Hubbard, in press-b). Similarly, the terms representational gravity, representational friction, and representational centripetal force abstractly and meta-

A
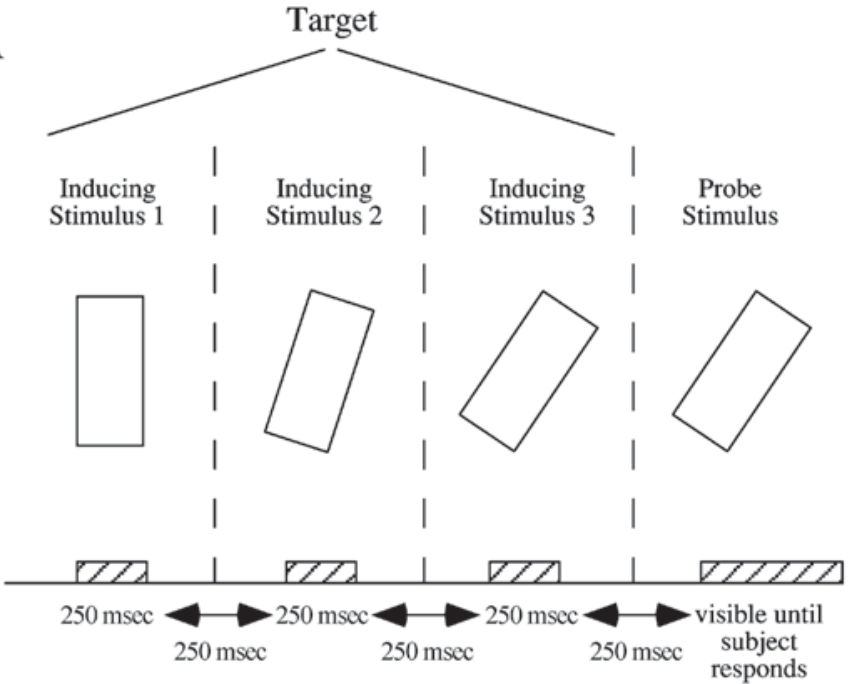

B

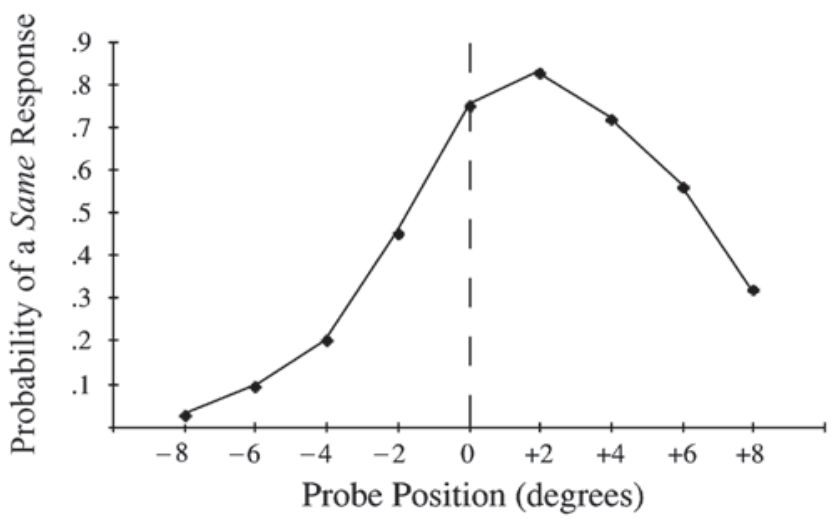

Figure 1. Experimental methodology and results from Freyd and Finke (1984). (A) A typical trial in which three inducing stimuli and a probe are presented. (B) The probability of a same response as a function of probe orientation relative to the final inducing stimulus. The dashed line in panel B is the true-same orientation of the final inducing stimulus; negative probes were rotated backward from the orientation of the final inducing stimulus by the indicated number of degrees, and positive probes were rotated forward from the orientation of the final inducing stimulus by the indicated number of degrees. Representational momentum is indicated by the higher probability of a same response to positive probes. 
phorically describe components of displacement consistent with how physical gravity, friction, and centripetal force would influence a physical object.

\section{Displacement in the Direction of Motion (Representational Momentum)}

The initial demonstration of a forward displacement in memory for the final position of a previously viewed moving target was provided by Freyd and Finke (1984; see panel A in Figure 1), who presented observers with computer-animated displays consisting of three concentric sequential presentations of a static rectangular target, referred to as the inducing stimuli, and a fourth static rectangle, referred to as the probe. The orientation of each inducing stimulus within a trial varied, and in some trials a consistent clockwise or consistent counterclockwise rotation was implied. The orientation of the probe was (1) rotated slightly backward from the orientation of the final inducing stimulus, (2) the same as the orientation of the final inducing stimulus, or (3) rotated slightly forward from the orientation of the final inducing stimulus. The observers judged whether the orientation of the probe was the same as or different from the orientation of the final inducing stimulus. If the inducing stimuli within a given trial implied motion in a consistent clockwise or consistent counterclockwise direction, the observers were more likely to respond same to probes rotated slightly forward than to probes rotated slightly backward (see panel B in Figure 1). Freyd and Finke (1984) suggested that this pattern resulted from memory for the orientation of the final inducing stimulus being displaced forward, and given that they hypothesized this displacement reflected the effects of implied momentum, they referred to the forward displacement as representational momentum.

Another early demonstration of a forward displacement in memory for the final position of a previously viewed moving target was provided by Hubbard and Bharucha (1988), who presented observers with computer animations of targets exhibiting smooth horizontal or vertical motion. The target vanished without warning, and after the target had vanished, the observers used a computer mouse to position the cursor at the display coordinates at which they judged the target to have vanished. The observers clicked a button on the mouse in order to record the display coordinates of the judged vanishing point, and differences between the judged vanishing point and the actual vanishing point along the axis of motion (the $x$-axis for horizontal motion and the $y$-axis for vertical motion, referred to as $M$ displacement) and along the axis orthogonal to motion (the $y$-axis for horizontal motion and the $x$-axis for vertical motion, referred to as $O$ displacement) were measured. Along the axis of motion, the observers were more likely to place the cursor slightly in front of the actual vanishing point, and this was consistent with the idea of representational momentum (displacement along the axis orthogonal to motion is not directly related to implied momentum and so will be considered later). Even though Freyd and Finke (1984) and Hubbard and
Bharucha used different methods of stimulus presentation and response collection, the results from these studies converged on the idea that memory for the final position of a moving target was displaced forward in the direction of target motion.

\section{Displacement Downward (Representational Gravity)}

Hubbard and Bharucha (1988) also reported that horizontally moving targets were displaced downward below the path of motion (in addition to being displaced forward along the axis of motion) and that descending targets exhibited larger forward displacement than did ascending targets (see Figure 2). Such patterns are consistent with observations that horizontally moving objects fall along a parabola, descending objects accelerate as they fall due to gravity, and ascending objects (that do not attain at least orbital velocity) decelerate as they rise. Hubbard (1990, 1995c, 1997) suggested that these patterns (and the downward component of displacement in general) were due to effects of implied gravity, and he referred to the effect of implied gravity as representational gravity. Hubbard (2001) reported that vertically moving targets that vanished high in the picture plane (i.e., descending targets
Leftward

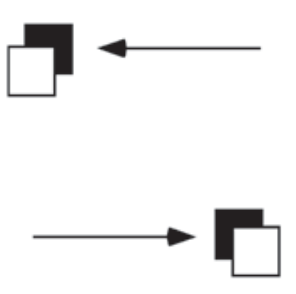

Rightward

\section{Ascending}

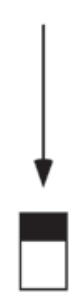

Descending

Judged vanishing point

Actual vanishing point
Figure 2. Effects of implied momentum and implied gravity on displacement of horizontal or vertical targets. The filled squares indicate the actual vanishing position, the open squares indicate the remembered vanishing position, and the arrows indicate the direction of target motion. Representational momentum is indicated by the forward displacement of remembered position, and representational gravity is indicated by the downward displacement for horizontal targets and by the larger forward displacement for descending targets than for ascending targets. From "Representational Momentum and Other Displacements in Memory as Evidence for Nonconscious Knowledge of Physical Principles," by T. L. Hubbard, 1998, in S. R. Hameroff, A. W. Kaszniak, and A. C. Scott (Eds.), Toward a Science of Consciousness II: The Second Tucson Discussions and Debates, p. 506. Copyright 1998 by MIT Press. Adapted with permission. 
that had fallen a shorter distance and ascending targets that had risen a longer distance) exhibited smaller forward displacement than did vertically moving targets that vanished low in the picture plane (i.e., descending targets that had fallen a longer distance and ascending targets that had risen a shorter distance), and this is consistent with observations that physical gravity acts to decrease the forward velocity of a rising object and increase the forward velocity of a falling object.

Evidence consistent with representational gravity has been found with several different methodologies. Munger, Solberg, and Horrocks (1999), Munger and Minchew (2002), and Munger and Owens (2004) found larger forward displacement for targets that rotated downward than for targets that rotated upward. Nagai, Kazai, and Yagi (2002) placed observers in an upright position or a prone (horizontal and facing downward) position and found that effects of implied gravity on displacement occurred only along the environmental axis aligned with gravitational attraction, regardless of the orientation of the observer (i.e., larger displacement for egocentric downward motion when the observers were upright and larger displacement for stimuli receding along the line of sight when the observers were prone). Evidence of representational gravity with a catching response was reported by McIntyre, Zago, Berthoz, and Lacquaniti (2001), who found that astronauts catching a ball in a $0-\mathrm{g}$ environment exhibited anticipatory catching responses more appropriate for catching a ball in a 1-g environment; it appeared as if representations of the ball's position (or of motor responses calibrated to the ball's position) automatically extrapolated or incorporated effects of gravity. Also, Ker-
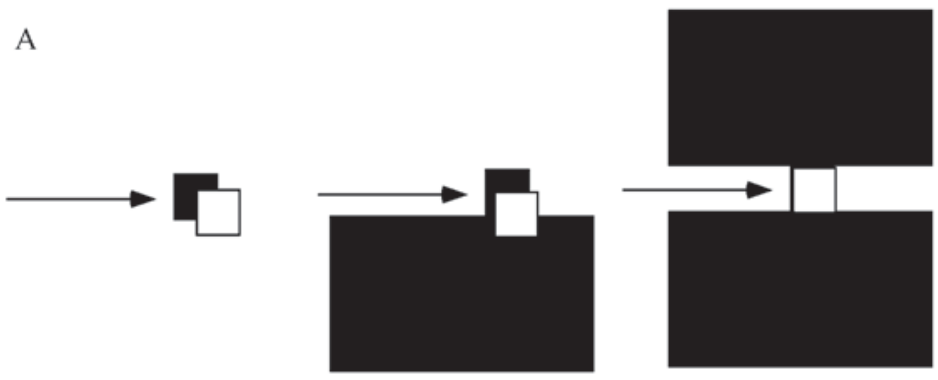

Blank Background

One Surface

Two Surfaces

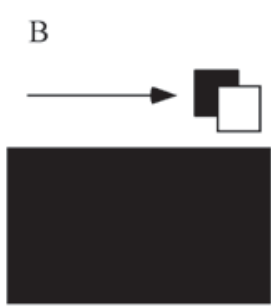

Above the Surface

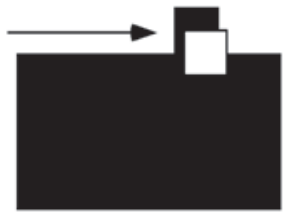

Along the Surface

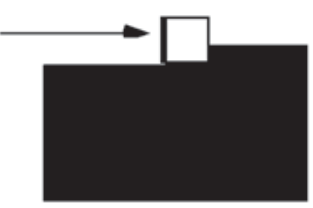

Compress the Surface

Judged vanishing point

Actual vanishing point

Figure 3. Effects of implied momentum and implied friction on displacement of a rightward target. The filled squares indicate the actual vanishing position, the open squares indicate the remembered vanishing position, and the arrows indicate the direction of target motion. (A) The target presented on a blank background, sliding across a larger stationary surface, or sliding between two larger surfaces. (B) The target separated from a larger surface, sliding across a larger stationary surface, or sliding along a larger surface and compressing that surface. In both panels, representational momentum is indicated by the forward displacement of remembered position, and representational friction is indicated by the decrease in forward displacement with increases in implied friction. From "Representational Momentum and Other Displacements in Memory as Evidence for Nonconscious Knowledge of Physical Principles," by T. L. Hubbard, 1998, in S. R. Hameroff, A. W. Kaszniak, and A. C. Scott (Eds.), Toward a Science of Consciousness II: The Second Tucson Discussions and Debates, p. 507. Copyright 1998 by MIT Press. Adapted with permission. 
zel, Jordan, and Müsseler (2001) replicated Hubbard and Bharucha's (1988) finding of a downward displacement for horizontally moving targets.

Hubbard (1995c) suggested that effects of representational gravity could combine with effects of representational momentum, and such a combination may be illustrated in a consideration of vertically moving targets: When representational gravity and representational momentum operated in the same direction (i.e., descending motion), they summed, and forward displacement was relatively large, whereas when representational gravity and representational momentum operated in opposite directions (i.e., ascending motion), they partially canceled out, and forward displacement was relatively small. Even so, the effects of implied gravity do not depend on the simultaneous presence of representational momentum, but are also found in memory for the position of stationary targets. Freyd, Pantzer, and Cheng (1988) presented observers with line drawings of a flowerpot that was depicted as resting on a table or as hanging from a hook, and when the observers were subsequently shown a drawing consisting of just the flowerpot, they were more likely to accept as same a drawing in which the flowerpot was presented a little lower in the display than a drawing in which the flowerpot was presented a little higher in the display. Consistent with this, Hubbard and Ruppel (2000) presented observers with a stationary square target, and memory for the target was displaced downward in the direction of implied gravitational attraction.

\section{Displacement After Contact \\ (Representational Friction)}

In early studies of displacement, the target was typically an isolated stimulus presented on a blank background. In a notable exception, Hubbard (1995b) presented observers with a horizontally moving target (1) on a blank background, (2) sliding across a single larger stationary surface, or (3) sliding between two larger stationary surfaces (see panel A in Figure 3). Forward displacement decreased as the number of stationary surfaces contacted by the target increased. A moving physical object that encountered a larger stationary surface would experience friction, and so the displacement pattern in Hubbard (1995b) is consistent with the notion that the momentum of a moving physical object decreases as that object encounters increased friction. Similarly, forward displacement was relatively large when the path of target motion was slightly separated from a larger stationary surface, decreased when the target slid along that surface, and decreased even more when the target slid along the surface and the surface behind the target appeared to be compressed (see panel B in Figure 3). Given the similarity of the decrease in forward displacement with increases in implied friction to the decrease in physical momentum of a moving object that encounters physical friction, Hubbard referred to the effect of implied friction as representational friction (Hubbard, 1995b, 1995c).
In one study that initially appeared to challenge the idea of representational friction, Cooper and Munger (1993) presented implied motion of geometric shapes that had a consistent surface area but varied in the amount of implied drag (e.g., a square that moved in the direction of one side or a triangle that moved in the direction of a point). Significant forward displacement was found for all the targets, but the amount of implied drag did not influence displacement. This pattern initially seemed inconsistent with the idea of representational friction, because a target with more implied drag would presumably experience more friction and should, thus, exhibit less forward displacement than would a target with less implied drag. However, Cooper and Munger's targets were presented on a blank background, and so the effects of friction or drag would have been between the target and the background medium the target was moving through, whereas Hubbard's (1995b) targets slid along a larger stationary surface, and so the effects of friction were between the target and another object. Given that a nontarget object is presumably more dense or rigid than the background medium, the effects of friction arising from contact with a nontarget object might be stronger or more salient than are the effects of friction arising from passage though a background medium.

Evidence consistent with representational friction has been found in several studies. Hubbard (1998b) reported a decrease in forward displacement of vertically moving targets with increases in the number of stationary surfaces contacted (i.e., with increases in implied friction). In a replication of Hubbard (1995b), Kerzel (2002b) reported a decrease in forward displacement when observers visually tracked a horizontally moving target that slid along the top of a larger stationary surface. In Hubbard (1995b, 1998b) and in Kerzel (2002b), the effects of implied friction may be viewed as combining with the effects of implied momentum, and as the effects of implied friction increased, the effects of implied momentum were partially canceled out. Hubbard (1995c) suggested that the effects of representational friction could combine with the effects of representational gravity, and such a combination is consistent with Bertamini's (1993) finding that memory for a stationary target on an inclined plane was displaced down the plane when the slope of the plane was relatively steep, but not when the slope of the plane was relatively shallow. With steeper slopes, implied friction between the target and the plane was relatively weaker than implied gravity, and so the target was displaced down the plane, whereas with shallower slopes, implied friction between the target and the plane was relatively stronger than implied gravity, and so the target was not displaced.

\section{Displacement Inward From a Curved Trajectory (Representational Centripetal Force)}

Hubbard (1996b) reported that memory for a target moving along a circular orbit was displaced forward along the tangent to the orbit and inward toward the focus of that 
orbit (see Figure 4). Forward displacement was consistent with momentum along the tangent and so was consistent with representational momentum. Inward displacement was consistent with the influence of centripetal force on a physical object moving along a circular trajectory, and so Hubbard (1996b) referred to the inward displacement as representational centripetal force. Furthermore, the magnitudes of forward displacement and of inward displacement increased with increases in angular velocity and with increases in radius length. A similar forward and inward displacement for targets moving along a circular orbit has been reported by Kerzel (2003b). Jordan, Stork, Knuf, Kerzel, and Müsseler (2002) and Müsseler, Stork, and Kerzel (2002) have reported forward displacement along the orbit for a target moving clockwise along a circular orbit. Freyd and Jones (1994) presented a target stimulus that moved through a spiral tube, and after exiting the tube, the target moved along a straight, curved, or spiral path before vanishing. Although Freyd and Jones did not measure separate forward and inward components of displacement, they reported that displacement along the path of motion was largest for targets that continued along a spiral path after exiting the tube, and such a pattern is consistent with the effects of representational momentum and representational centripetal force on an extrapolation of trajectory (see Hubbard, 1996b).

\section{PART II \\ Variables Influencing Displacement}

Since the initial reports on representational momentum appeared, a number of researchers have examined a wide range of influences on displacement. Hubbard (1995c) reviewed four major categories of influence on displacement: stimulus characteristics, implied dynamics and environmental invariants, memory averaging of the target and the context, and observer expectations. A large number of findings have appeared since that initial review, and consideration of these new data, in conjunction with previously known data, suggests a different and
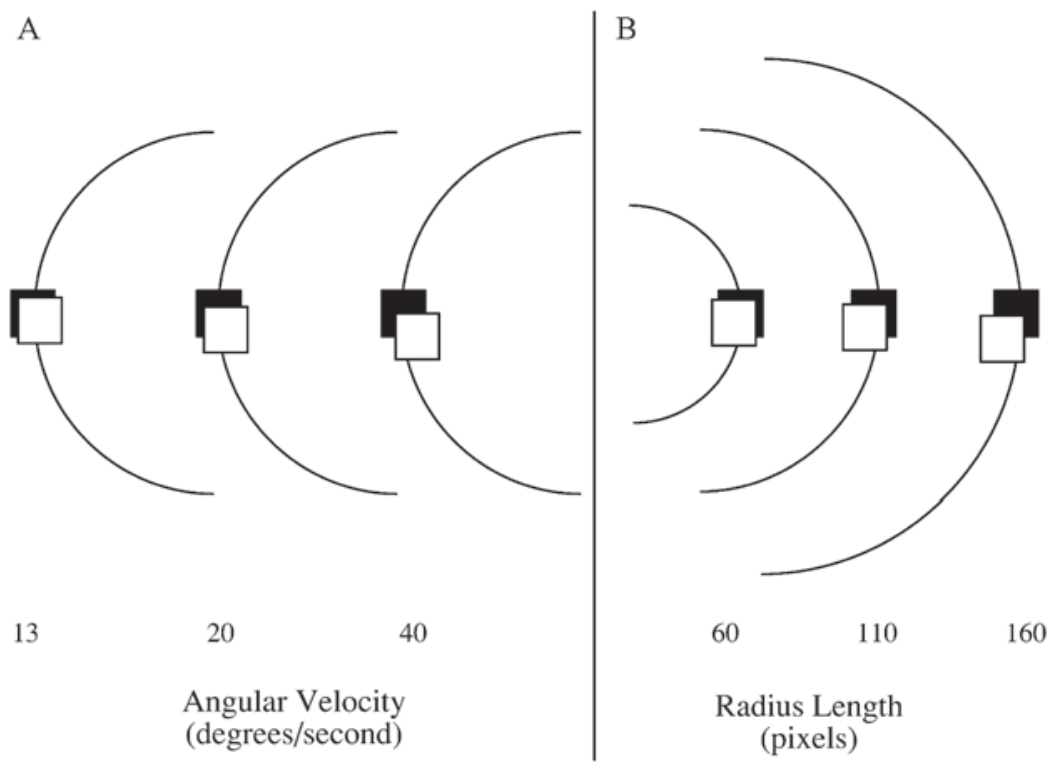

\section{Judged vanishing point \\ Actual vanishing point}

Figure 4. Effects of implied momentum and implied centripetal force in targets moving along a circular path. The filled squares indicate the actual vanishing position, the open squares indicate the remembered vanishing position, and the arrows indicate the direction of target motion. (A) Displacements for three different angular velocities for counterclockwise targets. (B) Displacements for three different radii for clockwise targets. In both panels, representational momentum is indicated by the forward displacement of remembered position along the tangent, and representational centripetal force is indicated by the displacement inward toward the center of the circular orbit. From "Representational Momentum and Other Displacements in Memory as Evidence for Nonconscious Knowledge of Physical Principles," by T. L. Hubbard, 1998, in S. R. Hameroff, A. W. Kaszniak, and A. C. Scott (Eds.), Toward a Science of Consciousness II: The Second Tucson Discussions and Debates, p. 508. Copyright 1998 by MIT Press. Adapted with permission. 
more theoretically neutral way of considering influences on displacement: characteristics of the (1) target, (2) display, (3) context, and (4) observer.

\section{Characteristics of the Target}

Some of the characteristics of the target that might potentially influence displacement in memory for the final position of a previously viewed target include the velocity and direction of target motion, the identity and shape of the target, the size of the target, whether the target depicts an animate or an inanimate stimulus, and the modality of the target. This category of variables is the most widely investigated of the potential variables that influence displacement and contains many of the stimulus characteristics reviewed in Hubbard (1995c), as well as additional variables not addressed in that earlier review.

Target velocity. Freyd and Finke (1985) varied the velocity of implied target rotation and found that faster implied velocities led to larger forward displacement (see also Munger \& Minchew, 2002); similarly, Hubbard and Bharucha (1988; Hubbard, 1990) reported that faster velocities for horizontally or vertically translating targets led to larger forward displacement. Finke, Freyd, and Shyi (1986) reported that forward displacement increased for targets undergoing acceleration and decreased for targets undergoing deceleration (see also Poljansek, 2002), even when the final velocity of the target was held constant. The effect of target velocity is one of the most robust influences on displacement in the direction of motion, although this effect is diminished (1) with increases in implied friction (Hubbard, 1995b), (2) if the target is initially stationary and its subsequent motion is attributed to contact from a moving object (Hubbard \& Ruppel, 2002), (3) at very high target velocities (Munger \& Owens, 2004), and (4) for continuous motion visual targets when observers cannot track the target (Kerzel et al., 2001). Similar effects of velocity have been reported for displacement in memory for auditory pitch (Freyd et al., 1990), although the interpretation of a possible velocity effect on displacement in memory for auditory pitch is questionable, given the use of linear (rather than logarithmic) spacing of inducing stimuli and probes in those studies (see Hubbard, 1995a).

Direction of target motion. Effects of the direction of motion for targets translating in the picture plane have consistently been found. Hubbard and Bharucha (1988; Hubbard, 1990) reported that horizontal motion led to larger forward displacement than did vertical motion and, as was noted earlier, that descending motion led to larger forward displacement than did ascending motion. Also, Hubbard (1990) reported that oblique motion led to forward displacement intermediate to that of horizontal motion and vertical motion. This ordering of displacement magnitude as a function of the direction of target motion has been found when the visible display was circular, longer along the horizontal axis, or longer along the vertical axis and so does not reflect the shape of the display. Although differences between horizontal and vertical mo- tion and between ascending and descending motion have been consistently reported, differences between leftward and rightward motion have not been reported as consistently. Halpern and Kelly (1993) reported that rightward motion resulted in larger forward displacement than did leftward motion, and they speculated that this resulted from asymmetries in visual processing in the cerebral hemispheres (see also Kerzel, 2003a). However, Hubbard (1990, 1995b; Hubbard \& Bharucha, 1988) and Cooper and Munger (1993) did not find consistent differences between displacement for leftward motion and displacement for rightward motion.

The effects of the direction of motion for targets appearing to translate in depth are mixed. Hubbard (1996a) manipulated apparent depth by varying the size of the target in the picture plane, and observers were instructed that changes in size reflected how a target would appear as it approached or receded. For receding targets, memory exhibited forward displacement consistent with representational momentum; for approaching targets, memory for slower targets exhibited backward displacement, whereas memory for faster targets exhibited forward displacement. Nagai et al. (2002) had observers wear polarizing lenses that allowed stereoscopic views of targets that appeared to approach or recede along the observers' line of sight. Both approaching and receding motion resulted in forward displacement, but forward displacement was larger for receding motion. In Hubbard (1996a) and in Nagai et al., forward displacement was larger with receding motion, and this pattern is consistent with a potential combination of representational momentum and boundary extension (see Hubbard, 1996a, in press-b). Hayes, Sacher, Thornton, Sereno, and Freyd (1996) presented stereoscopic images that implied approaching or receding motion along the observers' line of sight, and they reported forward displacement for approaching targets and for receding targets. Thornton and Hayes (2004) and Munger, Owens, and Conway (2005) reported forward displacement along the line of sight when stimuli suggested forward motion of the observer's viewpoint through a scene.

A consistent forward displacement has been found for targets that rotate in depth. Munger, Solberg, Horrocks, and Preston (1999) presented cube-shaped stimuli that rotated around different axes, and they reported that forward displacement was exhibited in all cases; however, if the axis of rotation was centered within the cube, there were no effects of direction of rotation on displacement, whereas if the axis of rotation was different from an axis of the cube, effects of direction of rotation were exhibited. Munger, Solberg, and Horrocks (1999) presented shaded figures of three-dimensional objects, and they reported that implied rotation around an axis that corresponded with both the viewer's and the object's coordinate systems led to larger displacement than did rotation around an axis that corresponded with neither the viewer's nor the object's coordinate systems. Munger and her colleagues suggested that the differences in displacement patterns for rotation in depth and for translation in depth might reflect 
the use of different cortical processing mechanisms for rotation and for translation. It is also possible that these differences might relate to differences in the changes of proximal size or average distance of the target; translation along the line of sight is strongly linked to changes in proximal size and average distance, whereas rotation in depth is less strongly linked to changes in proximal size or average distance.

The effects of the direction of motion for targets that rotate within the picture plane are mixed. A consistent direction of motion within a trial is necessary in order for forward displacement to be exhibited. Freyd and Finke (1984) reported that if the inducing stimuli on a given trial implied rotation in a consistent clockwise or consistent counterclockwise direction, forward displacement was exhibited, but if the inducing stimuli on a given trial did not imply rotation in a consistent clockwise or consistent counterclockwise direction, forward displacement was not exhibited. Kerzel (2002c) pointed out that in many previous studies, direction of rotation was blocked, and he reported that forward displacement was greatly diminished if direction of rotation varied within blocks (i.e., varied from trial to trial). However, robust forward displacement has been reported when the direction of rotation (Munger, Solberg, \& Horrocks, 1999; Munger, Solberg, Horrocks, \& Preston, 1999) or translation (Hubbard, 1990; Hubbard \& Bharucha, 1988) varied randomly within observers and across trials within a single block, and so forward displacement does not depend solely on across-trial expectancies of motion in a specific direction. A consistently larger forward displacement for either clockwise or counterclockwise rotation is usually not reported (e.g., Freyd \& Johnson, 1987; Kelly \& Freyd, 1987), but as was noted earlier, targets that rotate downward typically exhibit larger forward displacement than do targets that rotate upward (Munger \& Minchew, 2002; Munger \& Owens, 2004).

The effects of the direction of motion for targets that move along a circular or curvilinear path within the picture plane are mixed. Freyd and Jones (1994) presented targets that moved through a spiral tube and then followed a straight, curved, or spiral path upon exiting the tube. In two of three experiments, there was no influence of whether the target moved through a clockwise or counterclockwise spiral tube on the forward displacement of that target, and in the third experiment, targets that moved through a clockwise spiral tube exhibited larger displacement than did targets that moved through a counterclockwise spiral tube. Hubbard (1996b) presented targets that moved clockwise or counterclockwise along a circular orbit, and forward displacement (along the tangent of the orbit) was not influenced by target direction. Although Kerzel (2003b) presented both clockwise and counterclockwise motion around a circular orbit, the analysis appeared to collapse across direction. Although not involving curvilinear motion per se, Joordens, Spalek, Razmy, and van Duijn (2004) presented targets that moved along the perimeter of a rectangular clockface, and forward displacement was larger for targets that moved clockwise than for targets that moved counterclockwise. A consistent larger forward displacement for either clockwise or counterclockwise motion is usually not reported, but when a difference is found, clockwise motion typically results in larger forward displacement than does counterclockwise motion.

Target identity and shape. Kelly and Freyd (1987) reported that if each inducing stimulus within a trial was a radically different shape (e.g., rectangle, hourglass, or triangle), observers perceived a series of different static objects at different orientations, rather than a single object changing in orientation, and so forward displacement was not exhibited. If a consistent shape was used for all inducing stimuli within a trial, observers were more likely to perceive a single target in motion, and so forward displacement was exhibited. Differences in the internal markings of inducing stimuli also influenced displacement: When each of the inducing stimuli within a trial had a different pattern of internal markings, accuracies of probe judgments did not provide evidence of displacement, but reaction times of probe judgments were consistent with representational momentum (i.e., observers required more time to reject probes beyond the final target orientation than to reject probes behind the final target orientation). Kelly and Freyd suggested that differences in the internal markings were insufficient to completely overrule the apparent assumption of observers that different inducing stimuli were views of the same target at different orientations, but nonetheless, differences in the internal markings did weaken forward displacement. Thus, the occurrence of a robust forward displacement in memory for the target on any given trial requires that a constant target identity be maintained during that trial.

The effects of a specific identity label attached to a target have been examined. Reed and Vinson (1996) presented a target that ascended, and forward displacement was larger if the target was verbally labeled a rocket than if the target was verbally labeled a cathedral. Reed and Vinson suggested that this revealed an effect of conceptual knowledge on displacement; the observers knew that rockets were more likely to ascend than were cathedrals, and the increased likelihood of ascent for rockets than for cathedrals resulted in larger displacement for targets labeled as rockets than for targets labeled as cathedrals. Consistent with this, Kerzel (2003a) suggested that larger forward displacement on rocket trials in Reed and Vinson reflected an increased likelihood for the observers in the rocket trials to attend to a location further along the potential trajectory than did the observers in the cathedral trials. Displacement is also sensitive to differences between specific objects within a single category. Vinson and Reed (2002) examined whether object-specific effects were produced by conceptual context (e.g., knowledge that rockets ascend), visual features (e.g., whether the tip of the rocket was round or pointed), or an interaction of con- 
ceptual context and visual features, and they suggested that object-specific effects are most likely to influence displacement if a target is prototypical of its category and its identity corresponds to an object with a strong typical motion (see also Nagai \& Yagi, 2001).

In several studies of displacement, whether targets were pointed and whether target motion was in the direction of the point have been varied. Freyd and Pantzer (1995) presented observers with a moving target shaped like an arrow, and forward displacement was larger if the arrow moved in the direction of its point than if the arrow moved in the direction opposite to its point. Nagai and Yagi (2001) presented targets that varied in pointedness and in whether target motion was in a typical or an atypical direction (e.g., an airplane that moved in the direction of its nose exhibited motion in a typical direction, whereas an airplane that moved in the direction of its rudder exhibited motion in an atypical direction) and found evidence of a weak pointedness effect. Vinson and Reed (2002) found that pointedness increased displacement of a rocketlike shape, although they argued that prototypicality of shape was more important than was pointedness in evoking objectspecific effects. Cooper and Munger (1993) did not find effects on displacement of differences in implied drag of square or triangular translating targets, and given that the stimuli that differed in implied drag also differed in pointedness, the lack of an effect of implied drag suggested that pointedness did not influence displacement. In general, there might be an effect of pointedness when targets are depictions of familiar physical objects, but such an effect is weak and often is confounded with other influences.

Displacement in memory for shape has also been examined. Although not originally interpreted as representational momentum (because it predated introduction of that term), an overshooting of curvature consistent with representational momentum was reported by Foster and Gravano (1982), who found that the change in curvature of an illusory form undergoing apparent motion from a curved line to a straight line overshot the change in curvature (i.e., the straight line appeared to be curved in the opposite direction). Kelly and Freyd (1987) presented inducing stimuli that changed in shape. If inducing stimuli consisted of rectangles that widened or thinned, forward displacement in memory for shape was exhibited; however, if the inducing stimuli consisted of rectangles that changed into a square, forward displacement in memory for shape was not exhibited. It was suggested that forward displacement in memory for shape did not occur in the latter case, because the square was a prototypical or good ending point for a transformation of shape (and thus, no continuation of motion would have been extrapolated). Kelly and Freyd also found forward displacement in memory for the size of a square that increased or decreased in size across inducing stimuli (see also Hubbard, 1996a; White, Minor, Merrell, \& Smith, 1993), and so the lack of displacement when inducing stimuli were rectangles changing into a square did not result from a general lack of displacement for square stimuli but, rather, from the type of change across the inducing stimuli.
Target mass and size. Given that the momentum of a physical object is defined as the product of that object's velocity and mass, it could be predicted that the representational momentum of a target should be influenced by the implied velocity and by the implied mass of that target. As was noted earlier, effects of velocity on displacement in the predicted direction have often been found; however, effects of mass on displacement are not as clear. Cooper and Munger (1993) presented pyramid-shaped targets that rotated in depth, and the implied mass of the targets varied across trials but did not influence displacement. Hubbard (1995b) presented horizontally moving targets that varied in size across trials, and target size did not influence displacement along the axis of motion, although larger targets were displaced downward a larger distance along the orthogonal axis. Hubbard (1997) examined the effects of target size and implied mass on displacement of horizontally or vertically moving targets; for horizontal motion, target size influenced displacement along the axis orthogonal to motion, but not along the axis of motion, whereas for vertical motion, target size influenced displacement along the axis of motion, but not along the axis orthogonal to motion. Kozhevnikov and Hegarty (2001) presented ascending targets that varied in size across trials, and memory for smaller ascending targets exhibited larger forward displacement than did memory for larger ascending targets.

The findings regarding the effects of target size and implied mass on displacement initially seem inconsistent, but careful consideration reveals a pattern: Effects of target size are exhibited only along the axis aligned with implied gravitational attraction. If displacement in a direction not aligned with implied gravitational attraction is measured, target size does not influence displacement, whereas if displacement in the direction aligned with implied gravitational attraction is measured, larger targets exhibit larger downward displacement, regardless of the direction of target motion (for ascending targets, a smaller forward displacement is exhibited in memory for larger targets, and this is consistent with the general notion that larger targets are remembered as being lower in the display). Given that visually larger objects are usually perceived to be heavier (i.e., the size-weight illusion; Koseleff, 1957; for a review, see Jones, 1988), this pattern suggested that displacement was influenced by the implied weight of the target, rather than by the implied mass of the target. Weight is experienced along the axis aligned with gravitational attraction (weight $=$ mass $\times$ acceleration due to gravity) and reflects the subjective experience of mass for observers whose lifetimes have been spent within a gravitational field of constant strength (as found on the surface of the Earth). The idea that displacement reflects weight rather than mass suggests that displacement reflects subjective aspects of physical principles, rather than objective physical principles per se.

Target animacy. The pattern of motion a stimulus exhibits can be influenced by whether that stimulus is animate or inanimate, ${ }^{1}$ and so it is possible that displacement of a target could be influenced by whether that tar- 
get represents an animate or an inanimate stimulus. Freyd and Miller (1992) reported larger forward displacement in memory for a drawing of an abstract "creature" when the creature moved in the direction its "head" appeared to face than when the creature moved in the opposite direction, but no such difference in forward displacement was found for a control stimulus composed of the rearranged features of the creature. Halpern and Kelly (1993) did not find consistent differences in forward displacement in memory as a function of whether targets were drawings depicting animate (e.g., a rhinoceros or a fox) or inanimate (e.g., a truck or a motorcycle) stimuli. However, in Freyd and Miller and in Halpern and Kelly, both animate targets and inanimate targets were portrayed by computer-generated drawings, and so targets depicting animate referents were actually inanimate. The motions of targets depicting animate referents and of targets depicting inanimate referents were identical, and so real-world differences between animate motion and inanimate motion could not be exploited by the observers. Freyd and Pantzer (1995) compared forward displacement in memory for single static drawings of an arrow, a fish, and an airplane; although memory for the arrow produced forward displacement, neither memory for the airplane nor memory for the fish exhibited significant forward displacement.

Only a few studies of displacement have presented biologically appropriate or realistic motion. Thornton (1998) presented videotape clips of human faces beginning to smile or frown, and he reported that memory for the final facial expression was displaced backward toward a more neutral expression. However, the extent to which changes in facial expression involve momentum per se is questionable. Also, there is a clear maximum extent of facial expression, and the observers presumably anticipated a return to a more neutral expression, rather than an increasing intensity of the current expression beyond the maximum biological extent. Verfaillie, De Troy, and Van Rensbergen (1994) presented point-light walker figures, and in some conditions, transsaccadic memory for the figures was consistent with a representational momentumlike extrapolation from the posture of the walker at the initiation of a saccade to the posture of the walker at the completion of that saccade. Verfaillie and Daems (2002) presented observers with an animated sequence of a humanlike figure performing some action, and then the observers viewed a static picture of a humanlike figure and judged whether the picture portrayed a possible or an impossible pose. When the static picture presented a possible pose that was an extension of the movement of the just-viewed animated sequence, the observers were faster in their judgments, and this priming effect is consistent with representational momentum in memory for the actions (or postures) depicted in the animated sequence.

Auditory targets. Although the majority of studies on displacement have examined memory for visual stimuli, a small number of studies have examined displacement in memory for auditory stimuli. Kelly and Freyd (1987) and Freyd et al. (1990) reported forward displacement in memory for auditory pitch, and Freyd et al. (1990) reported effects of velocity and acceleration in memory for auditory pitch that were similar to the effects of velocity and acceleration previously reported for memory for visual targets. Hubbard (1995a) found that forward displacement in memory for auditory pitch was exhibited only if the duration of the final pitch was held for a sufficient length of time, and he also found consistent trends for larger forward displacement for descending pitches than for ascending pitches. Johnston and Jones (in press) presented a series of tones that implied a periodic cycle of ascending and descending motion in pitch space, and displacement in memory for the final pitch was (1) backward when the final pitch was at the highest or lowest pitch in the cycle (i.e., about to reverse direction) and (2) forward when the final pitch was not at the highest or lowest pitch in the cycle. Getzmann, Lewald, and Guski (2004) presented an acoustic target (continuous noise or noise pulses) that moved in the frontal horizontal plane. Listeners pointed to the final spatial position of the acoustic stimulus, and their judgments were displaced forward in the direction of motion (see also Getzmann, 2005).

\section{Characteristics of the Display}

Some of the characteristics of the display that might potentially influence displacement in memory for the final position of a previously viewed target include the surface form of a stimulus, the latency between when observers expect the target to vanish and when the target actually vanishes, the duration of the retention interval between when the target vanishes and when the observer responds, how the response of the observer is measured, and whether the observer receives error feedback regarding judgment of the target. Although Hubbard (1995c) discussed effects of retention interval on displacement, other variables in this category were not addressed in that earlier review.

Surface form. The surface form of a target refers to the format in which the target is presented. The two most common formats of target presentation in studies of representational momentum involve (1) a sequential series of stationary discrete stimuli that imply motion of a single target (e.g., Freyd \& Johnson, 1987; Munger, Solberg, Horrocks, \& Preston, 1999) or (2) a single target that appears to be undergoing smooth continuous motion (e.g., Hubbard, 1990; Hubbard \& Bharucha, 1988). ${ }^{2}$ Forward displacement is found with both types of surface forms, but across-study comparisons of whether these two types of surface form result in different magnitudes of displacement are difficult, because studies in which this issue has been examined have presented different types of motion (e.g., visual rotation, translation, or curvilinear motion; changes in auditory frequency). Evidence that whether a target exhibits implied motion or continuous motion might not influence displacement has been shown in two studies. Hubbard (1995a) did not find an effect on displacement in memory for auditory pitch as a function of whether the target was presented as a series of discrete inducing 
auditory frequencies or as a single continuous frequency glide. Munger and Owens (2004) did not find an effect on displacement in memory for the orientation of a visually presented rod as a function of whether the target was presented as a series of discrete inducing stimuli at different orientations or as a continuously rotating stimulus.

Evidence that whether a target exhibits implied motion or continuous motion might influence displacement has been found in three studies. Faust (1990) presented a horizontally moving target and found that forward displacement was larger with continuous motion than with implied motion. Poljansek (2002) did not find a main effect of the surface form of a visual target on displacement but did find an interaction of surface form with whether targets accelerated, decelerated, or maintained a constant velocity: Displacement was slightly smaller for continuous motion than for implied motion when motion decelerated, but it was slightly larger for continuous motion than for implied motion when motion accelerated. Kerzel (2003c) presented a target that moved along a circular trajectory, and the durations of the inducing stimuli and the interstimulus intervals (ISIs) between the inducing stimuli were varied. Kerzel (2003c) reported that forward displacement was smaller with shorter durations of inducing stimuli and ISIs - that is, forward displacement decreased as motion became smoother and more continuous. Overall, the data on whether a target exhibits implied motion or continuous motion might influence forward displacement in memory for the final position of that target are inconclusive, since some investigators (e.g., Munger \& Owens, 2004) have found equivalent displacement with implied motion and with continuous motion, whereas other investigators (e.g., Kerzel, 2003c) have found differences in displacement with implied motion and with continuous motion; furthermore, the differences that have been reported are not consistent.

A third format of target presentation in studies of representational momentum involves a single stationary stimulus that implies movement. Freyd (1983) presented frozenaction photographs drawn from a longer motion sequence (e.g., waves crashing on a beach), and observers judged whether a subsequently presented probe photograph was the same as or different from the original photograph. Probes drawn from slightly later in the sequence required more time to reject than did probes drawn from slightly earlier in the sequence. Futterweit and Beilin (1994) presented frozen-action photographs and nonaction (e.g., a still life or a person standing still) photographs. The observers were slower and less accurate when probes for frozen-action photographs were drawn from slightly later in the sequence than when probes were drawn from slightly earlier in the sequence, but no such asymmetries in responding were found with nonaction photographs. Freyd et al. (1988) reported that memory for the location of a target in a static scene was displaced in the direction of the implied forces, and Freyd and Pantzer (1995) reported that memory for the location of a triangle was displaced in the direction the triangle appeared to point.
However, Hubbard and Courtney (in press) reported that memory for the orientation of a T'ai-chi tu (i.e., yin-yang) figure was displaced in the direction opposite to implied rotation, and Hubbard and Blessum (2001) reported that memory for angular size was displaced in the direction opposite to implied expansion.

All of the types of surface form discussed so far involve a relatively passive observer who has no control of the target (i.e., involve exafferent stimuli). A fourth format of target presentation involves a more active control of the target by the observer (i.e., involves reafferent stimuli). Jordan and Knoblich (2004) presented a horizontally moving target that moved back and forth, and observers controlled the motion of the target by pressing arrow keys (i.e., pressing the ">" key would accelerate a rightwardmoving target or decelerate a leftward-moving target, and pressing the " $<$ " key would accelerate a leftward-moving target or decelerate a rightward-moving target). In an individual condition, each observer had control of both arrow keys; in a group condition, each observer had control of only one arrow key, and a different observer had control of the other arrow key. The target vanished at an uncued location, and forward displacement of a target exhibited by a given observer decreased with increases in the control that the observer had over target motion. Displacement can also be influenced by whether an observer has control over just the vanishing point of the target; Jordan et al. (2002) have reported that observers who pressed a button to trigger offset of a fast velocity target exhibited nonsignificant displacement, whereas observers who viewed a fast velocity target that vanished without warning exhibited backward displacement.

Latency to vanish. The majority of studies of displacement have presented a passive observer with a target that vanished at an uncued position (e.g., Hubbard \& Bharucha, 1988) or vanished at a highly predictable time (e.g., after the third inducing stimulus; Freyd \& Finke, 1984). In a notable exception, Jordan et al. (2002) asked observers to press a button to initiate disappearance of the target. The target traveled a circular path, and the observers were instructed to press a button to stop movement of the target after the target had traveled at least $90^{\circ}$ but before the target had traveled $360^{\circ}$. The target vanished 0 , 53, or 107 msec after the observer's buttonpress, and after the target had vanished, there was a 500-msec retention interval. Forward displacement decreased with increases in the latency between the buttonpress and when the target vanished. Jordan et al. suggested that this pattern occurred because displacement reflected activation of an action plan (to press the button to stop the target) that remapped perceptual space in light of expectations that the target would stop when the button was pressed. In other words, the remembered final position of the target corresponded to the position of the target at the time of the buttonpress (i.e., the intended vanishing position), rather than to the position of the target at the time that the target actually vanished, and so forward displacement decreased as the distance between the intended vanishing position and the 
actual vanishing position increased (i.e., decreased with increases in latency).

Retention interval. Finke and Freyd (1985) presented patterns of dots in which each dot exhibited implied motion in a different direction, and forward displacement reached an asymptote within $500 \mathrm{msec}$ and remained relatively stable. In a subsequent study, Freyd and Johnson (1987) presented implied motion of a rotating target; forward displacement peaked after a few hundred milliseconds, but instead of remaining stable as in Finke and Freyd (1985), forward displacement then decreased. They suggested that differences between their data and the data in Finke and Freyd (1985) resulted from the different sensitivities of the probes in the two studies; in Freyd and Johnson, a wide range of probe positions was used, and this provided a fine-grained measure of displacement, whereas in Finke and Freyd (1985), a narrow range of probe positions (a single forward position and a single backward position) was used, and this might have provided too coarse a measure of displacement. The effect of retention interval on forward displacement reported by Freyd and Johnson was replicated by Kerzel (2002c) when direction and starting position were blocked, but not when direction and starting position were randomized. Halpern and Kelly (1993) presented horizontal implied motion, and the forward displacement observed when the retention interval was $125 \mathrm{msec}$ was not significantly different from the forward displacement observed when the retention interval was $500 \mathrm{msec}$.

Finke and Freyd (1985), Freyd and Johnson (1987), and Halpern and Kelly (1993) presented observers with implied motion. Kerzel (2000) presented observers with continuous motion, and he reported that forward displacement increased until it reached an asymptote after approximately $250 \mathrm{msec}$ and then remained high. This pattern is consistent with those in Finke and Freyd (1985) and Halpern and Kelly but is not consistent with that in Freyd and Johnson. One possibility is that forward displacement resulting from horizontal (as in Halpern \& Kelly, 1993; Kerzel, 2000) or from horizontal and vertical (as in Finke $\&$ Freyd, 1985) translation does not decline as rapidly as does forward displacement resulting from rotation (as in Freyd \& Johnson, 1987). Translation (in which there is a clear change in target location) might result in a stronger sense of motion than does rotation (in which there is not a clear change in target location), and so forward displacement might be stronger or take longer to decay for translation than for rotation; thus, it is possible that an eventual decline in displacement might have been observed if displacement had been measured after a longer retention interval in Kerzel (2000) or in Halpern and Kelly. Also, given that translation and rotation appear to be processed by different neural areas (see, e.g., Sakata, Kusunoki, \& Tanaka, 1993), it is possible that forward displacement resulting from translation could exhibit different temporal properties than would forward displacement resulting from rotation.

The initial increase in displacement in Freyd and Johnson (1987) and in Kerzel (2000) might seem inconsistent with the initial decrease in displacement with increases in latency between the buttonpress and when the target vanished in Jordan et al. (2002). However, an initial increase in displacement might have occurred during the 500-msec retention interval in Jordan et al., and so evidence of an initial increase in displacement might have been found with smaller retention intervals. Also, none of the observers in Jordan et al. reported noticing differences between the latency conditions, and this is consistent with the suggestion noted earlier that the observers encoded the final target position as reflecting the position of the target at the time of the buttonpress, rather than the position of the target at the time the target actually vanished. In such a case, though, the actual retention interval on a given trial would reflect the sum of the latency for the target to vanish and the 500-msec retention interval, and this would yield de facto retention intervals of 500, 553, and 607 msec. Kerzel (2000) did not examine displacement after retention intervals longer than $500 \mathrm{msec}$, but a decrease in displacement over a 500- to 600-msec range of retention intervals appears to be consistent with Freyd and Johnson. Thus, the displacement pattern in Jordan et al. appears to be consistent with the possibility that forward displacement declines after 500 msec.

In the majority of the studies in which the effects of retention interval on displacement have been examined, a probe judgment response measure has been used (for an exception, see Jordan et al., 2002), and this might be because it is straightforward to manipulate retention interval with such a methodology: Simply vary the latency between the offset of the final inducing stimulus or a smoothly moving target and the onset of the probe. The effect of retention interval should, presumably, be independent of the response measure; however, use of a cursor-positioning response measure involves difficulties in separating the effects of retention interval per se from the effects of the time required to locate and appropriately position the cursor. Also, the time required to locate and appropriately position the cursor potentially could vary widely from trial to trial and could be longer than the brief duration of displacement suggested in some studies. It might be that observers determine the display coordinates at which the target vanished, encode those specific coordinates, and only then locate and position the cursor. Such a strategy might offer one way in which representational momentum could be exhibited at the longer response latencies typical of cursor positioning, but resolution of this issue awaits further research. Also, the effects of retention interval on the displacement resulting from variables other than implied momentum or in directions other than the direction of motion have not been examined.

Response measures. Response measures in studies of displacement have usually involved either cursor positioning or probe judgment. In cursor positioning, observers use a computer mouse to position the cursor at the display coordinates at which the target vanished (although in a small number of cases, the cursor was positioned by use of arrow keys on the keyboard). By clicking a button on the mouse, the judged vanishing point is recorded, 
and that judged vanishing point is compared with the actual vanishing point to provide a direct measure of displacement. Cursor positioning involves recall and psychophysically reduces to the method of adjustment. In probe judgment, a stationary probe appears after the target has vanished, and observers judge whether the probe is at the same position as the previously perceived final inducing stimulus (or the same position at which a smoothly moving target vanished) or at a different position. Observers usually press one key to make a same response and a different key to make a different response. The position of the probe relative to the final position of the target is varied across trials, and the probabilities of same judgments to probes behind the final position, at the final position, or beyond the final position are measured and provide an indirect measurement of displacement. Probe judgment involves recognition and psychophysically reduces to the method of constant stimuli. Cursor positioning is typically used with continuous motion targets, and probe judgment is typically used with implied motion targets or frozen-action photographs, but response measure can be varied independently of surface form (e.g., as in Kerzel, 2003c).

There are advantages and disadvantages with cursor positioning and with probe judgment. Cursor positioning provides a more direct measurement of displacement and typically requires fewer trials than does probe judgment (e.g., with cursor positioning, a given vanishing point might be presented 5-10 times; however, with probe judgment, typically 5-9 probe positions are presented for each vanishing point, and each probe position must be presented multiple times in order to obtain a meaningful proportion of same responses for that probe position). A disadvantage of cursor positioning is that some stimulus dimensions might not be easily adapted to such a spatial measure (e.g., auditory frequency or visual brightness). Probe judgment provides better control of the retention interval and minimizes the motor components of the response. A disadvantage of probe judgment is that pilot testing or other experience is often necessary in order to determine an appropriate spacing of probe positions so that any hypothesized effects may be found (e.g., it is possible that the coarse spacing in Finke \& Freyd, 1985, could not capture the decay of displacement shown with the more fine-grained spacing in Freyd \& Johnson, 1987), and the placement of probes limits the types of displacement that can be observed (e.g., probes along the axis of motion of horizontally moving targets could not reveal effects of representational gravity).

In a small number of studies, displacement has been examined using reaching or grasping responses. Kerzel (2003c) reported that forward displacement for a target moving along a circular trajectory was larger with cursor positioning or reaching than with probe judgment, and Kerzel and Gegenfurtner (2003) reported that forward displacement for a horizontally moving target was larger with reaching than with probe judgments. Ashida (2004) reported that forward displacement for a horizontally moving target was larger with reaching when the hands were not visible than with reaching when the hands were visible or with cursor positioning. These patterns are generally consistent with previous speculation regarding the existence of separate perception-foridentification (as in probe judgment) and perception-foraction (as in reaching) pathways (e.g., Milner \& Goodale, 1995). Brouwer, Franz, and Thornton (2004) presented visual stimuli consisting of two spheres that moved toward or away from each other, and the spheres were either isolated (i.e., spheres) or connected by an adjoining rod (i.e., dumbbells). Changes in grasp aperture consistent with representational momentum occurred for dumbbells, but not for spheres, and visual judgments consistent with representational momentum occurred for dumbbells and weakly for spheres. Brouwer et al. suggested that whether a target translated or transformed influenced whether larger displacement was exhibited with visual responses or with grasping responses, but resolution of this awaits further research.

Error feedback. Freyd (1987) has suggested that representational momentum is impervious to error feedback (see also Finke \& Freyd, 1989). This claim was based on Finke and Freyd's (1985) study in which a verbal message of correct or error appeared in the display after observers made a same or different judgment regarding a probe. However, feedback was provided only during practice trials and was not provided during experimental trials, and feedback was relatively uninformative (e.g., feedback did not explicitly distinguish between false alarms and misses and did not indicate whether the probe had been behind, the same as, or beyond the final inducing stimulus). Finke and Freyd (1985) concluded that feedback did not have an effect on representational momentum, because (1) error rates in practice trials and in experimental trials were the same and (2) the observers' error rates did not improve across experimental trials, despite the observers' viewing the same pattern of inducting stimuli on every experimental trial. However, given the uninformative nature of feedback during practice trials and given that feedback was not provided during experimental trials, it is not clear how (explicit) learning could have occurred, because the observers would not have known how to correct their errors during practice trials and were unaware of their errors during experimental trials. Any conclusions regarding the effects of feedback on displacement must await future research.

\section{Characteristics of the Context}

Some of the characteristics of the context that might potentially influence displacement in memory for the final position of a previously viewed target include the presence of nontarget elements in the display, the relationship of the target to any nontarget elements in the display, expectations and knowledge regarding target behavior that arise in an observer as a consequence of viewing the target in a single trial or across multiple trials, and a priori expectations and knowledge regarding target behavior that an observer brings to the experimental setting. As these 
examples suggest, context can be either physical (e.g., nontarget stimuli in the display) or cognitive (e.g., expectations based on previous experience with the target), and this category of variables includes influences from the memory-averaging and the observer expectancy categories reviewed in Hubbard (1995c), as well as additional variables not addressed in that earlier review.

Surrounding context. Hubbard (1993b) presented a rectangle undergoing implied rotation, and the rectangle was surrounded by a larger concentric square frame. The frame was visible during presentation of (1) the inducing stimuli but not the probe, (2) the probe but not the inducing stimuli, or (3) both the inducing stimuli and the probe. If the frame was stationary but positioned beyond the orientation of the final inducing stimulus or if the frame rotated in the same direction as the inducing stimuli, forward displacement in memory for the target increased. If the frame was stationary but positioned behind the orientation of the final inducing stimulus or if the frame rotated in the direction opposite to the rotation of the inducing stimuli, forward displacement in memory for the target decreased. Favretto (2002; see also Favretto, Hubbard, Brandimonte, \& Gerbino, 1999) presented a stationary target that increased or decreased in luminance during each trial, and luminance of the background surrounding the target varied across trials. Memory for the final luminance of the target was displaced backward (see also Brehaut \& Tipper, 1996), but backward displacement (1) increased when targets on a darker background increased in luminance or when targets on a lighter background decreased in luminance and (2) decreased when targets on a darker background decreased in luminance or when targets on a lighter background increased in luminance. In Hubbard (1993b) and in Favretto, displacement of the target was partly biased toward or in the direction of the surrounding context.

Similar effects of context have been found when the context only partially surrounded the target or when the target moved between elements of context. Whitney and Cavanagh (2002) presented two bars that moved horizontally in opposite directions, and above and below each bar were translating gratings that moved in the same direction as the adjacent bar or in the opposite direction. If the gratings moved in the same direction as the bar, memory for the bar was displaced forward, and displacement was larger for faster velocities. If the gratings moved in the direction opposite to the bar, forward displacement was reduced. As in Hubbard (1993b) and Favretto (2002), displacement of the target in Whitney and Cavanagh was biased in the direction of the surrounding context. Gray and Thornton (2001) presented a horizontally moving target that approached a barrier, and the target was on an otherwise blank field or moved between two rows of briefly flashed or continuously visible stationary distractors. The presence of stationary distractors greatly decreased forward displacement (see also Kerzel, 2002a). Also, the magnitude of forward displacement correlated positively with the magnitude of underestimation of the time to collision of the target with the stationary barrier, and this is consistent with the possibility that underestimation of time to collision might be related to forward displacement of the target.

In many studies of the effects of surrounding context on displacement, the context surrounding the target has not been as rich as that which typically occurs in everyday experience. In a notable exception, Thornton and Hayes (2004) presented observers with videotape clips (filmed from a stationary viewpoint) that were drawn from longer sequences of real-world scenes (e.g., a train station, a shopping area, or a town square) in which there were multiple stimuli in motion. Probes consisted of single frames from the videotape that were drawn from before the end of the clip, the end of the clip, or after the end of the clip. The observers were more likely to accept as same the probes from after the end of the clip than the probes from before the end of the clip, and this suggests that representational momentum occurs for stimuli in surrounding contexts that are ecologically rich and typical of everyday experience. Interestingly, this displacement occurred even though the clips contained multiple potential targets, many of which were moving in different directions, and so previous findings that displacement could occur in memory for multiple target displays (e.g., Finke \& Freyd, 1985; Finke et al., 1986; Finke \& Shyi, 1988) were replicated. These findings strengthen confidence in (1) the validity of laboratory investigations of displacement in which relatively impoverished stimuli have been used, and (2) the potentially widespread and ecological nature of displacement.

Although many studies of the effect of surrounding context on displacement have focused on movement of the target through that context, whether displacement occurs when an observer's viewpoint (i.e., when the observer) moves through the surrounding context has also been examined. Munger et al. (2005) presented observers with examples of movement of an observer's viewpoint forward through (1) an Egyptian scene depicting a pyramid in a desert setting or (2) a Mexican scene depicting a pyramid in a forest and mountain setting. Significant forward displacement of the observer's viewpoint occurred for both scenes. Thornton and Hayes (2004) placed observers in a virtual reality environment in which those observers were passengers in a vehicle moving along a straight road, and on either side of the road were buildings, trees, and other landmarks. At an uncued location, a 250 -msec blank interval was inserted into the virtual reality animation. On some trials, the animation resumed at the same location at which it had stopped, and on other trials, the animation resumed at an earlier or later location. The observers judged whether the location at which the animation resumed was the same as the location at which it had stopped and were more likely to accept as same a location from slightly later than a location from slightly earlier. The results from Munger et al. (2005) and from 
Thornton and Hayes suggest that representational momentum occurs for motion of the self (i.e., the observer's viewpoint), as well as for motion of an external target.

The surrounding context need not consist of nontarget stimuli visible within the display but could, instead, be supplied in a top-down manner by activation of relevant schematic structures. Hubbard (1993a) presented listeners with an ascending sequence of three auditory frequencies that corresponded to the root, the fifth, and the octave of a major scale ( $d o, s o l, d o$ of the $d o, r e, m i$ scale). The octave was either slightly flattened (i.e., lowered in frequency from a true octave), in tune, or slightly sharpened (i.e., raised in frequency from a true octave). If memory for the final pitch reflected representational momentum and was not influenced by the musical structure of the stimuli, memory for the final pitch should have been displaced forward in all conditions. Alternatively, if the sequence of auditory frequencies activated listeners' schemata regarding musical intervals and displacement was influenced by that cognitive context, memory for the final pitch should have been displaced forward when the final pitch was flattened from a true octave and displaced backward when the final pitch was sharpened from a true octave. The displacement pattern was more consistent with a schematic activation of musical knowledge than with representational momentum, thus supporting the notion that the cognitive context within which a target is embedded influences displacement.

Landmarks. The examples of context thus far have been more "global," in the sense that they (at least partially) surrounded the target and provided a larger framework within which the target was embedded. However, context may also be more "local," in the sense of providing a single object or landmark that provides a point of reference to one side of the target. Hubbard and Ruppel (1999) presented a target that moved toward or away from a larger stationary landmark. Forward displacement of the target increased when that target moved toward the landmark and decreased when that target moved away from the landmark. Representational momentum appeared to combine with a landmark attraction effect (i.e., with a bias in the remembered or perceived position of a target toward a landmark: see, e.g., Bryant \& Subbiah, 1994) to determine the displacement of the target; if landmark attraction and representational momentum operated in the same direction (i.e., if the target moved toward the landmark), they summed, and forward displacement was relatively large, whereas if landmark attraction and representational momentum operated in opposite directions (i.e., if the target moved away from the landmark), they partially canceled out, and forward displacement was relatively small. Also, a moving target that passed to one side of a larger stationary landmark was displaced toward that landmark (Hubbard \& Ruppel, 1999), and a stationary target near a larger stationary landmark was displaced toward that landmark (Hubbard \& Ruppel, 2000).

In accord with these results, Kerzel (2002a) reported that memory was displaced toward a briefly flashed stim- ulus that was presented at the moment the target vanished or shortly thereafter. Kerzel (2002a) questioned use of the term landmark for such nontarget stimuli; indeed, in the case in which a nontarget stimulus is briefly flashed, landmark does not seem to be appropriate, and Kerzel's suggestion of distractor may be more appropriate. However, if a nontarget stimulus is present during the entire presentation of the target (as in Hubbard \& Ruppel, 1999), use of landmark might be more appropriate. As Kerzel (2002a) suggested, the sudden appearance of a distractor at the moment the target vanished or shortly thereafter might have drawn attention away from the (previous) location of the target and toward the distractor (and so memory for the target was displaced toward the distractor); even so, it is not clear that a similar shift of attention away from the (previous) location of the target and toward a landmark would necessarily have occurred at the time the target vanished or shortly thereafter if that landmark had been visible during the entire presentation of the target. Conversely, Munger and Owens (2004) found that a distractor that appeared at the moment the target vanished and that was aligned with the final position of the target increased forward displacement of the target, and they suggested that this occurred because a flash-lag effect would result in an increase in the subjective distance between the distractor and the target.

In Hubbard (1995b), memory for a horizontally moving target was displaced a larger distance downward along the orthogonal axis if the target slid over a single larger stationary surface than if the target was on a blank background. This might reflect representational gravity if the target was on a blank background and a combination of representational gravity and landmark attraction if the target was above a larger stationary surface. Displacement along the orthogonal axis was larger if a single larger stationary surface was below the target than if a single larger stationary surface was above the target. This might reflect a combination of representational gravity and landmark attraction; if representational gravity and landmark attraction operated in the same direction, they summed, and displacement was relatively large, whereas if representational gravity and landmark attraction operated in opposite directions, they partially canceled out, and displacement was relatively small. If a horizontally moving target slid between larger stationary surfaces above and below that target, displacement along the orthogonal axis was greatly reduced, since landmark attraction from each of the surfaces canceled out. An analogous pattern was found in Hubbard (1998b), in which a vertically moving target was displaced along the orthogonal axis toward a single larger stationary surface on either the left or the right side of the target, but if larger stationary surfaces were on both the left and the right sides of the target, displacement along the orthogonal axis did not differ from zero.

Expectations regarding future target motion. Hubbard and Bharucha (1988) presented a horizontally or vertically moving target within a larger stationary square frame, and the target collided with and bounced off the 
frame several times before the target and the frame simultaneously vanished. If the target vanished after having just bounced off the frame, memory was displaced forward, and this was consistent with representational momentum. However, if the target vanished just before colliding with the frame or at the moment of collision, memory was displaced backward, as if the observer had anticipated an impending bounce, and the backward displacement was larger if the target vanished at the moment of collision than if the target vanished just before collision. In a related study, Verfaillie and d'Ydewalle (1991) measured displacement of targets that exhibited motion in a single direction or motion in an oscillatory pattern. If displacement for a target at the end of a single direction of motion was measured, forward displacement occurred, but if displacement for a target at the edge of an oscillation (at the moment when the target would have been expected to reverse direction) was measured, forward displacement did not occur. Similarly, Johnston and Jones (in press) presented auditory stimuli that oscillated in pitch. When the final pitch was at the edge of an oscillation (i.e., pitch motion was about to reverse direction), memory for that final pitch was displaced backward, whereas when the final pitch was not at the edge of an oscillation, memory for that final pitch was displaced forward.

In studies in which target motion oscillated, there was a clear boundary beyond which target motion did not pass. A similar influence of a boundary beyond which target motion does not pass can also be seen if a target does not oscillate. Hubbard and Motes (2002) presented a translating target on an otherwise blank background. As was expected, memory for the final position exhibited robust forward displacement. Memory for the initial position was also measured and, in accord with Actis-Grosso and Stucchi (2003; Actis-Grosso, Stucchi, \& Vicario, 1996) and Thornton (2002), exhibited robust backward displacement. However, when Hubbard and Motes (2005) enclosed identical target motion within a larger stationary frame, outside of which the target was never visible, this additional physical context reversed the displacement pattern (i.e., there was robust forward displacement in memory for the initial position and backward displacement in memory for the final position) when the initial position and final position of the target were adjacent to the frame. The boundaries defined by the frame might have provided a limit on the range of visible target motion and, thus, a limit on displacement. An analogous pattern was observed when Müsseler et al. (2002) had observers judge initial position or final position when a small dot flashed near the initial position or the final position; the location of the flashed dot might have provided a boundary on target motion and, thus, a limit on displacement.

Hubbard (1994) presented observers with a horizontally or vertically moving target that contacted a stationary barrier (i.e., a line orthogonal to the path of motion), and the target either bounced off or crashed through the barrier. Prior to the appearance of the target on each trial, observers received a verbal cue informing them whether that trial would be a bounce trial or a crash trial. Forward displacement of the target was larger in bounce trials than in crash trials, as if an elastic bouncing off the barrier preserved target momentum and a rigid crashing through the barrier depleted target momentum. Of greater interest, in some conditions invalid cues were occasionally presented (e.g., a cue of crash was given on a trial in which the target would actually bounce), and forward displacement was decreased in invalid cue trials relative to valid cue trials. A decrease in forward displacement in invalid cue trials initially seems paradoxical, because the observers were actually more accurate in judgments of final target position in invalid cue trials, but this might reflect (1) a lack of extrapolation in the actual target direction or (2) a canceling out of extrapolation in the expected (invalid) direction by perceptually driven extrapolation in the actual direction. Regardless, the results of Hubbard (1994), as well as those of Hubbard and Bharucha (1988), Verfaillie and d'Ydewalle (1991), and Johnston and Jones (in press), suggest that displacement reflects the embedding

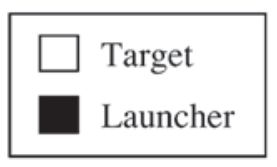

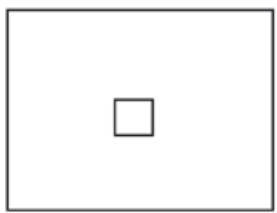

Target Stationary, Launcher Not Visible

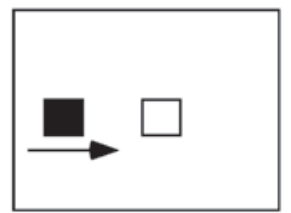

Launcher Enters and Approaches Target

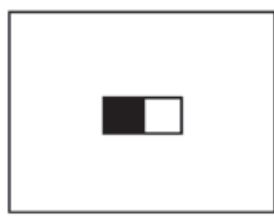

Launcher Contacts Target, Stops

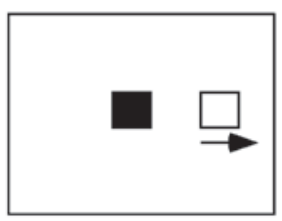

Launcher Stationary, Target Moves Away

Figure 5. Illustration of Michotte's launching effect. A stationary target is shown.A moving launcher then enters from one side of the display and contacts the target. At the moment the launcher contacts the target, the launcher becomes stationary, and the target begins to move. (See also Hubbard \& Ruppel, 2002.) 
of target motion within the context defined by a larger event structure.

Even though displacement reflects expectations regarding future target motion, displacement cannot be attributed to a conscious prediction of a subsequent target position (e.g., observers' predicting the location of the next potential inducing stimulus, rather than reporting memory for the last inducing stimulus). Finke and Freyd (1985) presented implied motion targets, and probes could match the position of (1) the final inducing stimulus, (2) the next inducing stimulus (had the inducing sequence continued), or (3) the previous (penultimate) inducing stimulus. No differences in the error rates or response times between probes at the position of the next inducing stimulus and probes at the position of the previous inducing stimulus were exhibited. Finke and Shyi (1988) clustered probe positions around the final inducing stimuli, which was referred to as the memory task, or clustered probe positions around the next configuration of inducing stimuli had the inducing sequence continued, which was referred to as the extrapolation task. ${ }^{3}$ In the memory task, a standard forward displacement was exhibited, whereas in the extrapolation task, a marginally significant backward displacement was exhibited. Similar differences between displacement in a memory task and displacement in an extrapolation task were found by Munger and Minchew (2002), who presented three-dimensional renderings of "three-armed figures" undergoing implied rotation; furthermore, Munger and Minchew also found that the magnitude of backward displacement in the extrapolation task increased with increases in target velocity.

Attribution of the source of target motion. Hubbard, Blessum, and Ruppel (2001) examined displacement of stationary targets that began to move after being contacted by a moving object. Their displays were based on Michotte's (1946/1963) launching effect paradigm (see Figure 5); in the launching effect, the motion of a previously stationary target is attributed to a moving object, referred to as the launcher, that contacts the target, but only if each of the values of several parameters (e.g., latency between when the launcher contacted the target and when the target began moving, direction of subsequent target motion relative to previous launcher motion, and ratio of previous launcher velocity and subsequent target velocity) is within a narrow range. Hubbard et al. (2001) reported that the forward displacement of launched targets was less than the forward displacement of nonlaunched control targets (e.g., less than isolated targets of the same velocity and direction of motion that were presented in an otherwise blank display). In general, displacement was influenced by attributions regarding the source of target motion: Displays modeled on those previously shown to induce a launching effect (in which target motion was attributed to the launcher) resulted in a decrease in forward displacement of the target, whereas displays modeled on those previously shown not to induce a launching effect (in which target motion was perceived to be more autono- mous) did not result in a decrease in forward displacement of the target.

The importance of the launcher in the (mis)localization of a launched target was demonstrated by findings that the forward displacement of a launched target (1) is influenced by the velocity of the launcher and (2) decreases with increases in the distance traveled by the target after launching (Hubbard \& Ruppel, 2002). Furthermore, decreases in displacement typical of a target in a launching effect display are not exhibited if the final position of the launcher and the initial position of the target are spatially separated and the gap between the launcher and the target is not bridged by a visible intermediary (Hubbard \& Favretto, 2003). It is not necessary for the launcher to actually move, because illusory gamma movement triggered by the sudden appearance of a stationary launcher adjacent to a target that then immediately moved away from the launcher can result in a decrease in displacement similar to that observed with a launching effect display (Hubbard, Ruppel, \& Courtney, 2005). The notion that displacement can be influenced by attributions regarding the source of target motion is consistent with findings noted earlier that displacement is influenced by embedding target motion within the context defined by a larger event structure. Also, findings that the magnitude of displacement tracks attributions of causality provided the first behavioral evidence convergent with the introspective reports of Michotte's (1946/1963) observers and, thus, demonstrated that displacement can be a useful tool for the study of other cognitive phenomena (see also Hubbard, 2004).

\section{Characteristics of the Observer}

Some of the characteristics of the observer that might potentially influence displacement in memory for the final position of a previously viewed target include various strategies, behaviors, and demographic variables. The influences of this category of variables on displacement have received relatively little empirical study, and possible influences on displacement of a number of potential strategic or demographic variables have not been investigated. Although Hubbard (1995c) mentioned the single study on the effects of age on displacement available at that time, other variables in this category were not addressed in that earlier review.

Attention. Hayes and Freyd (2002) presented a dot that moved leftward or rightward above a square that increased or decreased in size, and the allocation of attention was manipulated by varying the probability of a probe's appearing for the dot or for the square (e.g., the dot was considered to be less attended in a condition in which $20 \%$ of the probes were for the dot and $80 \%$ of the probes were for the square). Forward displacement in memory for the location of the dot increased when less attention was allocated to the dot. In a second experiment, observers counted aloud in time with the ticking of a metronome while viewing the dot; this dual-task condition resulted in 
larger displacement of the dot than did a single-task condition in which the observers just viewed the dot. Hayes and Freyd suggested that attention was necessary for stopping the automatic extrapolation of target position (i.e., for stopping displacement). Munger and Owens (2004) presented a rotating rod, and in some conditions, a cubeshaped distractor aligned with the final orientation of the rod flashed immediately before the rod vanished. Forward displacement of the rod was larger when the cube was presented than when the cube was not presented. Although displacements were consistent with an attention-based account, variances were not consistent with an attentionbased account; as was noted earlier, Munger and Owens suggested that the larger forward displacement they observed when the cube was flashed occurred because the participants perceived a gap between the flashed object and the moving target because of a flash-lag effect.

Joordens et al. (2004) presented a small target that moved around the perimeter of a rectangular clockface. At a noncued time during the trial, the color of the clockface changed, and the target continued to move for another 500-800 msec before it vanished. Observers in the single-task condition or in the dual-task condition pressed a designated key as soon as they detected the color change and then used cursor positioning to indicate the location of the target at the time the color change occurred; in addition, the observers in the dual-task condition simultaneously listened to an extended string of spoken numerical digits (ranging from 1 to 9) and had to respond each time three odd digits in a row were presented. For clockwise motion, the target exhibited no displacement in the single-task condition and forward displacement in the dual-task condition; for counterclockwise motion, the target exhibited backward displacement in the single-task condition and no displacement in the dual-task condition. The results for clockwise motion are consistent with the effects of divided attention on representational momentum found by Hayes and Freyd (2002), but Joordens et al. have suggested that differences in displacement between single-task and dual-task conditions for both clockwise and counterclockwise motion reflected a combination of representational momentum and an opposite-acting compensation effect, and not attention per se.

Kerzel (2003a) presented vertically moving inducing stimuli, and a distractor was flashed in front of, behind, or to the side of the final target position after the target had vanished. Presentation of the distractor resulted in a significant backward displacement in memory for the final position of the target, and this backward displacement was larger if the distractor was in front of the target than if the distractor was behind the target. Kerzel proposed that attention was necessary for maintaining extrapolation of target position and for generating displacement; however, this view is opposite to that of Hayes and Freyd (2002). Müsseler et al. (2002) presented a target traveling a circular trajectory, and a stationary stimulus was flashed when the target vanished. If the flashed stimulus was relevant to the task (i.e., if the observers were instructed to judge target position at the time of the flash), backward displacement in memory for the final position of the target occurred, whereas if the flashed stimulus was irrelevant to the task (i.e., if the observers were instructed to ignore the flash and judge the final position of the target), a weak forward displacement occurred. Müsseler et al. did not directly compare displacement in an irrelevant flash condition with displacement when a flash was not presented, but an inspection of their data suggests that forward displacement in an irrelevant flash condition was less than forward displacement when no distractor was presented.

In Hayes and Freyd (2002), Joordens et al. (2004), and Munger and Owens (2004), memory for the target exhibited larger forward (or smaller backward) displacement when a distractor was presented, whereas in Kerzel (2003a), memory for the target exhibited backward displacement when a distractor was presented. One possible explanation for this difference is that distractors were considered irrelevant for judgments of the target in Hayes and Freyd, Joordens et al., and Munger and Owens, but relevant in Kerzel (2003a); however, Kerzel (2003a) described distractors in his study as irrelevant. A second possible explanation is that distractors in Hayes and Freyd, Joordens et al., and Munger and Owens were present (albeit for different durations) while the target was still visible, whereas the distractor in Kerzel (2003a) flashed during the retention interval after the target had already vanished. It may be that a division of attention results in larger displacement while a target is visible, but once the target vanishes, the representation of the target is more fragile and a perceived distractor masks any forward extrapolation of the target that would otherwise have occurred. In the latter case, memory averaging toward previous positions of the target might occur, and the backward displacement observed by Kerzel (2003a) might be accounted for. However, it is not clear whether such an account would be consistent with the effect of flash relevancy in Müsseler et al. Whether decreases in attention allocated to a target increase or decrease displacement of that target and what variables modulate the effect of attention on displacement are not yet clear.

The findings of Hayes and Freyd (2002), Joordens et al. (2004), Kerzel (2003a), Munger and Owens (2004), and Müsseler et al. (2002) suggest that the allocation of attention can influence displacement. However, the reverse might also be true, because displacement might influence the allocation of attention. Kerzel et al. (2001) presented a horizontally smoothly moving target and then a probe, and the probe was slightly behind the final position of the target, at the final position of the target, or slightly beyond the final position of the target. The probe consisted of a circle that contained a gap at either the top or the bottom, and it was presented for approximately $10 \mathrm{msec}$. Observers judged whether the gap was at the top or the bottom of the probe, and they were more accurate if the probes were slightly beyond the final position of the target than if the probes were slightly behind the final position of the 
target. Kerzel (2003a) reported a similar facilitation for judgments of probes slightly beyond the position of the final inducing stimulus when implied motion of the target was presented. Of course, it is not completely clear from such examples whether a shift of attention results in displacement or whether displacement results in a shift of attention, and some researchers have even debated whether attention itself exhibits a form of momentum similar to representational momentum (e.g., Pratt, Spalek, \& Bradshaw, 1999; Snyder, Schmidt, \& Kingstone, 2001).

Eye movements. Although some researchers (e.g., Finke \& Freyd, 1985; Freyd \& Johnson, 1987) presented a fixation point prior to when the target appeared, such a fixation point usually vanished before the target appeared, and any subsequent eye movements were not monitored or controlled. In a notable exception, Kerzel (2000; Kerzel et al., 2001) compared displacement when observers fixated a stationary point some distance away from the target with displacement when observers visually tracked the target. If the observers visually tracked a smoothly moving target, their pursuit eye movements overshot the final position of the target, and forward displacement in memory for the final position of the target was exhibited, but if the observers fixated a stationary point some distance away from a smoothly moving target, forward displacement in memory for the final position of the target was decreased or even absent. However, such a fixation did not diminish forward displacement of a target undergoing implied motion (Kerzel, 2003a) or downward displacement of a horizontally moving target undergoing continuous motion (Kerzel et al., 2001). Ashida (2004) found that fixation did not influence displacement with reaching when the participants' hands were not visible but decreased displacement with reaching when participants' hands were visible and with cursor positioning. Also, whether observers visually track a moving sound source does not influence displacement for the location of that sound source (Getzmann, 2005).

Kerzel (2002b) replicated many of the earlier experiments of Hubbard, but with the addition of instructions for observers to visually track the target or to fixate a stationary point some distance away from the target and/or with monitoring of eye movements. One reason why Hubbard (1995c) had previously rejected an eye movementbased account of displacement is that displacement reflects the anticipated direction of target motion, rather than the actual direction of target motion at the time the target vanishes (e.g., as in the collision condition in Hubbard \& Bharucha, 1988), but Kerzel (2002b) pointed out that eye movement patterns can reflect an anticipated change of direction, and he found decreases in forward eye tracking prior to a change in target direction. If targets exhibited smooth motion across the top of a larger stationary surface (as in Hubbard, 1995b), the observers who fixated a corner of that larger stationary surface exhibited less forward displacement in memory for the target than did the observers who visually tracked the target. Kerzel (2003b) replicated the general forward displacement and inward displacement in Hubbard (1996b) in which targets followed a curved path but displacement was influenced by fixation: If the observers visually tracked the target, a forward displacement and a reduced inward displacement were found, but if the observers fixated the center of the circular path, a reduced forward displacement and a robust inward displacement were found.

Action plans. Studies of displacement usually have focused on sensory input and top-down cognitive influences and have not taken into consideration whether motor outputs or intentions influence displacement. The work of Jordan and colleagues is a notable exception. Jordan and Knoblich (2004) reported that whether observers had control over the direction and velocity of a target influenced displacement for that target, and this suggests that activation of action plans used in controlling a target can influence displacement of that target. Jordan et al. (2002) reported that displacement of a target was influenced by whether observers pressed a button to trigger offset of the target or the target vanished without warning, and this also suggests that activation of action plans related to controlling a target can influence forward displacement of that target. It might not even be necessary that activation of action plans involve a target or engagement with a target per se in order to influence displacement for that target; Jordan et al. have proposed that the backward displacement they observed when observers fixated a stationary fixation point away from a target, rather than visually tracked a smoothly moving target, was due to the generation of action plans relevant to fixation on the fixation point. However, it is not clear why action plans for fixation away from a target would apparently reduce forward displacement only for continuously moving targets and would not influence displacement for implied motion targets.

Jordan and colleagues (Jordan \& Knoblich, 2004; Jordan et al., 2002) did not conceive of action plans as limited to motor responses but, instead, conceived of action plans as involving a tight coupling of motor responses with perceptual space, such that actions are specified in terms of distal events (i.e., outcomes in the environment), rather than in terms of motor events (i.e., particular muscular actions). Such a view is consistent with Prinz's $(1992,1997)$ common coding theory, which suggests a sharing or overlap of the mechanisms used for perception and the mechanisms used for action planning. As a result of these shared or overlapping mechanisms, what an observer perceives (or represents) can be attenuated or modulated by motor actions that the observer is planning. Alternatively, perhaps the differences in displacement that Jordan et al. attributed to an activation of action plans simply reflect interference from a concurrent task (e.g., activating an action plan is analogous to flashing a distractor, in that both events divert attention or other cognitive resources or processes away from the target; cf. Kerzel, 2003a). Also, if observers decide a priori to press the button when the target reaches a specific location, a decreased or backward displacement might reflect an inaccurate prediction of when that target would arrive at the vanishing point to be produced and would not reflect memory per se; this would be consistent with Finke and 
Shyi's (1988) and Munger and Minchew's (2002) findings that observers who predicted the next position in a sequence of inducing stimuli exhibited negative displacement for the predicted position.

Age of the observer. There have been only two studies of displacement in children, and both studies focused on representational momentum. Futterweit and Beilin (1994) presented third-grade children, fifth-grade children, and adults with pairs of frozen-action photographs. If the photographs were shown in real-world temporal order, all the observers were more likely to respond that the second picture was the same as the first picture, and there were no differences in displacement as a function of age. Hubbard, Matzenbacher, and Davis (1999) presented first-grade children, fourth-grade children, and adults with small square targets that exhibited smooth horizontal or vertical motion, and displacement was measured with cursor positioning. All the observers exhibited forward displacement, and forward displacement was larger in the youngest children than in the adults. Given that Kelly and Freyd (1987) previously suggested that representational momentum relied on analogue representation, Hubbard et al. (1999) speculated that forward displacement was larger in the youngest children because those children may have relied on analogue representation more than did the adults. The youngest children in Hubbard et al. (1999) were younger than the youngest children in Futterweit and Beilin, and this difference might account for the different findings in the two studies. Alternatively, the continuous motion stimuli in Hubbard et al. (1999) may have been more effective than the frozen-action photographs in Futterweit and Beilin in suggesting motion to younger children.

Psychopathology. Jarrett, Phillips, Parker, and Senior (2002) examined representational momentum in patients diagnosed with schizophrenia, healthy schizotypic individuals, and a control group. Given the deficits in motion perception and in visual tracking that accompany schizophrenia, patients with schizophrenia and healthy schizotypic individuals had been predicted to exhibit smaller representational momentum than would the control group; however, there was a strong trend (in the opposite direction) for patients with schizophrenia and for healthy schizotypic individuals to exhibit larger representational momentum than did the control group. Jarrett et al. speculated that this trend reflected the failure of inhibition of automatic processes that is characteristic of individuals with schizotypic disorders. Conners, Wyatt, and Dulaney (1998) examined representational momentum in a group of patients diagnosed with mental retardation and in a control group, and although both groups exhibited representational momentum, the patient group diagnosed with mental retardation exhibited smaller representational momentum than did the control group. Conners et al. suggested that this pattern indicated that the observers with mental retardation processed motion in the same general way as did the observers without mental retardation, although the observers with mental retardation were less efficient in motion processing.
Physiology of displacement. The experience of displacement phenomena such as representational momentum is accompanied by activation at retinal, geniculate, and cortical levels. Berry, Brivanlou, Jordan, and Meister (1999) reported that firing of retinal ganglion cells anticipated the arrival of a continuously moving bar stimulus, and Sillito, Jones, Gerstein, and West (1994) reported that descending cortical information primed cells in the lateral geniculate nucleus for processing subsequent motion in a specific direction. White et al. (1993) presented inducing stimuli consisting of square shapes that varied in size and were located at a central fixation point, and probes were flashed in the left or right visual field. With the smallest retention interval $(500 \mathrm{msec})$, representational momentum (for changes in target size) was larger if the probes were presented in the left visual field than if the probes were presented in the right visual field, but this asymmetry vanished with longer retention intervals. Halpern and Kelly (1993) reported that forward displacement of horizontally moving targets was larger for targets presented in the left visual field. In accord with these asymmetries, Amorim et al. (2000) reported a magnetoencephalographic investigation in which greater cortical activity was exhibited by the right parietal cortex when observers exhibited representational momentum.

In fMRI investigations of representational momentum, Kourtzi and Kanwisher (2000) and Senior et al. (2000) reported that if observers were presented with still pictures that implied motion (i.e., frozen-action photographs), cortical areas such as V5/MT traditionally associated with the processing of motion information were activated. The importance of area V5/MT for representational momentum was also illustrated by an absence of representational momentum if a task previously shown to elicit representational momentum was performed immediately after transcranial magnetic stimulation of area V5/MT (Senior, Ward, \& David, 2002). Senior et al. (2000) also reported that the presentation of still pictures that implied motion resulted in increased activity in the anterior cingulate cortex. In an fMRI investigation, Rao et al. (2004) reported that observers engaged in an implied motion task based on Freyd and Finke's (1984) rectangle paradigm exhibited additional activity in the prefrontal cortex but no additional activity in MT/MST, relative to a control task. The findings of Kourtzi and Kanwisher, Senior et al. (2000), and Rao et al. suggest that different cortical structures may underlie representational momentum in different types of targets; furthermore, activation of cortical areas such as V5/MT, the anterior cingulate, and the prefrontal cortex is consistent with a role for high-level influences in representational momentum.

\section{PART III \\ Some General Empirical Conclusions}

The preceding discussion considered a wide range of influences on displacement. A number of specific empirical conclusions regarding displacement were drawn (e.g., forward displacement usually increases with increases in 
target velocity, displacement is toward or in the direction of surrounding context, target size and mass influence displacement only along the axis aligned with implied gravitational attraction, and displacement reflects the embedding of target motion within the context defined by a larger event structure), and a number of areas in which more research is needed before specific empirical conclusions can be drawn were noted (e.g., the effects of error feedback and the effects of the allocation of attention). In addition to the relatively specific conclusions regarding variables that influence displacement that were drawn in Part II, a number of more broadly based conclusions regarding displacement more generally can also be drawn, and these will be discussed in Part III.

\section{Does All Displacement Reflect the Same Underlying Cause?}

Researchers interested in displacement have typically focused on the effects of implied momentum and on representational momentum. Even so, there have been reports in which displacement was "backward" (e.g., luminance changes; Brehaut \& Tipper, 1996); in such cases, the direction of displacement was not consistent with the direction of stimulus change, and/or changes in the referent physical stimulus did not entail physical momentum, and so such displacement should not be said to reflect representational momentum. Similarly, displacement can occur in directions other than along the target trajectory (e.g., downward displacement of horizontally moving targets; Hubbard, 1990) and can occur with stationary targets (e.g., downward displacement of stationary targets; Freyd et al., 1988), and such displacement would not reflect momentum. Just as not all changes in or forces acting upon physical objects involve physical momentum, not all displacement involves representational momentum. Displacements of different targets can reflect different combinations of different influences, and representational momentum is just one type of influence. When considered at the level of specific influences and mechanisms, all displacement does not reflect the same underlying cause. However, when considered at a broader, more abstract, and more computational level, all displacement might reflect similar solutions to the same general type of problem, and this will be considered further in Part IV.

\section{Does Displacement Reflect Physical Principles?}

Even if a given displacement does not result solely from the effects of implied momentum, it might be suggested that all displacement nonetheless reflects some combination of implied physical principles. Indeed, in Part I, it was pointed out that some patterns of displacement were consistent with the effects of physical principles other than or in addition to momentum. The extent to which displacement reflects physical principles per se has been widely debated in the literature; theories of displacement suggest a variety of potential effects of physical principles ranging from an incorporation of the principle of momen- tum into mental representation (e.g., Finke et al., 1986) to a rejection of any internalization of physical principles (e.g., Kerzel, 2000, 2003a). The empirical evidence is clear that (1) displacement does not always correspond to predictions based on physical principles and (2) variables unrelated to physical principles (e.g., the presence of landmarks, target identity, or expectations regarding a change in target direction) can influence displacement. Rather than asking whether displacement reflects physical principles in an all-or-nothing fashion (i.e., whether forward displacement is completely dependent on an internalization of momentum or completely unrelated to any internalization of momentum), it might be more useful to consider the extent to which displacement might be influenced by implied physical principles and how implied physical principles might contribute to the overall displacement of a target.

\section{Does Displacement Reflect Naive Physics?}

Even if displacement does not reflect objective physical principles, it might be suggested that displacement reflects naive conceptions of physical principles. Several studies have suggested that the forward displacement of moving targets might reflect a notion of naive impetus (e.g., Hubbard \& Ruppel, 2002; Kozhevnikov \& Hegarty, 2001), and to the extent that displacement reflects naive impetus, displacement could be said to reflect naive physics. Similarly, studies within the naive physics literature have suggested the existence of a curvilinear impetus notion that influences judgments of naive observers regarding the potential trajectory of an object ejected from a curved or spiral tube (e.g., McCloskey \& Kohl, 1983), and it is possible that the choice of such a trajectory could reflect an averaging of a robust representational momentum and a decaying representational centripetal force (Hubbard, 1996b; but for a dissenting view, see Kerzel, 2003b). Findings that displacement is influenced by implied weight, rather than by implied mass, suggest that displacement might reflect subjective consequences of physical principles, rather than objective physical principles per se (Hubbard, 1997), and an emphasis on subjectivity is consistent with a more naive understanding of physical principles. Even so, information based on a naive understanding of physical principles or on subjective consequences of physical principles appears to be just one of many types of information that could potentially contribute to the displacement of any given target (for a more detailed discussion of the relationship of displacement and naive physics, see Hubbard, in press-b).

\section{Does Displacement Reflect an Artifact of Computer-Generated Stimuli?}

Studies of displacement have typically presented computer-generated stimuli that, lacking true mass or volume, would not experience the actual effects of physical principles such as momentum or gravity. However, rather than providing a criticism of studies of displace- 
ment, observations that computer-generated stimuli evoke responses more appropriate to solid three-dimensional physical objects illustrate just how strong biases in mental representation consistent with implied invariant physical principles actually are. Even when a target would not experience the effects of physical principles or does not exhibit appropriate changes in motion, the representation of target motion nonetheless responds as if that target did experience the effects of physical principles and did exhibit the appropriate changes in motion. ${ }^{4}$ Another potential concern with the use of computer-generated stimuli involves persistence of the display. If the monitor displaying computer-generated moving stimuli exhibited slow decay rates, perhaps a lingering image of the target might influence displacement. However, and as noted by Freyd and Finke (1984) and by Hubbard and Bharucha (1988), such an effect would operate in the direction opposite to target motion, and so forward displacement could not be caused by slow decay of the display. Similarly, slow decay rates would not produce displacement along the orthogonal axis or account for displacement in memory for a frozen-action photograph.

\section{Does Displacement Reflect Eye Movements?}

Hubbard (1995c) rejected an eye movement-based theory of representational momentum because forward displacement (1) occurs with auditory stimuli for which eye movements would be less relevant, (2) occurs with multitarget visual displays in which each target moves in a different direction and it is highly unlikely that eye movements could track the motion of every target, because that would entail moving the eyes in different directions simultaneously, and (3) reflects the anticipated, rather than the actual, direction of target motion. As was noted earlier, Kerzel (2002b) challenged Point 3 and reported that anticipatory eye movements anticipate a change in target direction, and he suggested that such anticipatory eye movements might account for forward displacement if observers anticipate that the target will change direction. However, such a notion seems to ignore that any anticipation that leads to anticipatory eye movements arises in other (possibly higher order) structures or mechanisms, and so eye movements would not be ultimately causal. Even so, Point 1 has been strengthened (e.g., Getzmann, 2005), Point 2 has been strengthened (e.g., Thornton \& Hayes, 2004), and a new point (4) may be added: Representational momentum occurs in memory for implied motion stimuli and in memory for frozen-action photographs, neither of which elicits pursuit eye movements. Although eye movements are related to forward displacement for the special case of a continuously moving visual target, they (even if coupled with visual persistence and foveal bias) cannot be the primary cause of representational momentum more generally, nor do eye movements seem to be related to other types of displacement (e.g., downward displacement attributed to representational gravity; Kerzel et al., 2001). ${ }^{5}$

\section{Does Displacement Reflect Modular Processes?}

Although displacement exhibits many qualities of a modular process (see Freyd, 1987), numerous findings suggest that displacement does not exhibit information encapsulation (i.e., impenetrability to an observer's knowledge, beliefs, and expectations; see, e.g., Hubbard, 1993a, 1994; Hubbard et al., 2001; Johnston \& Jones, in press; Reed \& Vinson, 1996; Verfaillie \& d'Ydewalle, 1991), which is a key component of modularity (for a discussion of modularity, see Fodor, 1983). Hubbard (1995c) concluded that the magnitude and direction of displacement were consistent with the influence of higher order knowledge of the target and the context or event within which the target was embedded, and even though this conclusion is still broadly true, displacement has subsequently been found to be uncorrelated with observers' explicit knowledge of physical principles (Freyd \& Jones, 1994; Kozhevnikov \& Hegarty, 2001). The apparent impenetrability of displacement to explicit physical knowledge is consistent with the possibility that at least some aspect of displacement is cognitively impenetrable and thus, perhaps, modular. Therefore, previous claims that displacement was or was not modular may have been overstated. Rather than asking whether displacement reflects a modular process, it might be more useful to consider which aspects of displacement can be influenced by different types of information and how both modular and nonmodular processes contribute to the overall displacement of a target (cf. Finke \& Freyd, 1989).

\section{Does Displacement Reflect High-Level Processes?}

There are several strands of evidence that displacement reflects high-level cognitive processes rather than low-level sensory processes. First, displacement is influenced by attributions regarding the source (e.g., Hubbard, 2004; Hubbard \& Favretto, 2003) and anticipated direction (e.g., Hubbard, 1994; Johnston \& Jones, in press; Verfaillie \& d'Ydewalle, 1991) of target motion. Second, displacement is influenced by target prototypicality and identity (Reed \& Vinson, 1996; Vinson \& Reed, 2002) and schematic context (e.g., Hubbard, 1993a). Third, representational momentum seems to be related to increased activity in several cortical regions (e.g., Amorim et al., 2000; Rao et al., 2004; Senior et al., 2000). Fourth, given that rotation may be processed in different cortical structures than is translation and that implied motion stimuli or frozen-action photographs might activate different cortical regions than do continuous motion stimuli, it is more parsimonious to suggest that a single or small number of high-level processes produce equivalent patterns of displacement across all the different types of stimuli. Fifth, given that forward displacement occurs for visual, auditory, and haptic aspects of a stimulus, it is more parsimonious to suggest a single or small number of high-level processes than a multiplicity of modalityspecific or dimension-specific low-level processes. Of 
course, a conclusion that displacement reflects high-level processes does not entail that low-level processes do not contribute to displacement, because top-down information from high-level processes could certainly influence low-level processes (e.g., expectations regarding target motion driving anticipatory eye movements).

\section{PART IV \\ Toward a Computational Theory of Displacement}

In seeking to understand a process such as displacement, it is useful to consider first whether that process serves some useful purpose or provides some benefit. Marr (1982) referred to this type of consideration as a computational theory about that process, and the salient consideration for a computational theory of displacement involves "what problem does displacement solve?" Once such a potential purpose or benefit of displacement has been articulated, questions regarding the representations and algorithms that lead to displacement (and the implementation of those representations and algorithms in specific models or structures) can be more meaningfully developed. Accordingly, one possible direction of a computational theory of displacement will be suggested in Part IV.

\section{Bridging the Gap}

Hubbard (1999, in press-b) explicitly suggested that displacement occurs because it aids in the spatial localization of physical objects and facilitates rapid motor responding to objects in the environment (see also Finke et al., 1986). Accurate spatial localization is important for calibrating an observer's response to a stimulus so that a maximally effective and adaptive interaction with that stimulus might be achieved. Consider the example of an observer trying to intercept a moving object. When a moving object is first sensed, it will be at a specific position $P_{1}$ in space (see Figure 6). This initial sensation will initiate a sequence of perceptual, cognitive, and perhaps motor processes. These processes are extremely fast, but they do take a minimum amount of time. During this time, a moving object does not pause motionless at position $P_{1}$, waiting for the observer's processing to be completed; rather, that object continues to move, and by the time the observer's processing is completed and an immediate motor response from the observer would reach the object, the object is at position $P_{2}$. If the response to a stimulus is
A

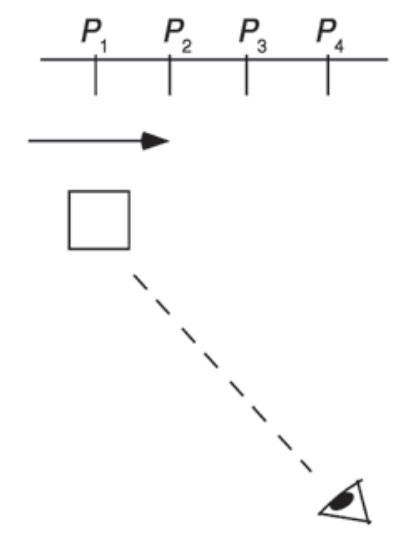

Observer Initially Senses Moving

Target and Initiates Perceptual/ Cognitive/Motor Processing. Target Located at $P_{1}$.
B Spatial Position
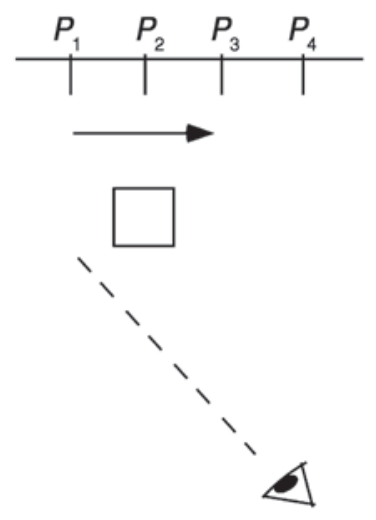

Observer Ready to Respond to

Moving Target. Perceptual/

Cognitive/Motor Processing

Complete. Target Located at $P_{2}$.

Figure 6. Illustration of the importance of displacement. (A) A moving target at spatial position $\boldsymbol{P}_{1}$ is initially sensed, and that begins a sequence of perceptual, cognitive, and motor processes. During this time, the target continues to move. (B) The initial perceptual and cognitive processing is complete, but the target is no longer at the position where it was initially perceived but has moved to spatial position $\boldsymbol{P}_{2}$. In order for a response, such as catching, blocking, hitting, or intercepting the target, to be maximally effective, an observer must compensate for the movement of the target from $\boldsymbol{P}_{1}$ to $\boldsymbol{P}_{2}$ and "bridge the gap" between the initially perceived position and where the target will be when a response from that observer reaches the target. This bridging of the gap is accomplished by representational momentum and related types of displacement. From "Bridging the Gap: Possible Roles and Contributions of Representational Momentum," by T. L. Hubbard, in press, Psicológica. Copyright 2006. Adapted with permission. 
to be maximally effective (e.g., if the person is to successfully intercept the moving object), that response should be tailored to the stimulus as that stimulus would be when the response reaches it at $P_{2}$, and not as that stimulus was when it was initially sensed at $P_{1}$. In other words, the gap between the initial perceived $P_{1}$ and the subsequent action $P_{2}$ needs to be bridged.

Providing a bridge between $P_{1}$ and $P_{2}$-that is, bridging the gap between perception and action-might be the problem that displacement solves. In other words, the purpose of displacement might be to predict $P_{2}$ of the target on the basis of the information at $P_{1}$. Displacement thus reflects an anticipation of where a target will be when an immediate response from the observer reaches that target, and this should enable a more effective or adaptive interaction with the target. Interestingly, such anticipatory effects of displacement parallel automatic anticipatory responses generated by some physiological reflex actions (e.g., removing a hand from a hot stove), in that both types of anticipations foster adaptive responding by bridging the gap between perception and action. ${ }^{6}$ Such a similarity suggests that displacement may be viewed as a representational reflex arc connecting sensory input and motor output. However, unlike behavior that results from a physiological reflex arc, displacement that results from a representational reflex arc can be modulated by additional information that could increase the effectiveness or adaptiveness of responding (e.g., if an observer expects a target to reverse direction, it would not be adaptive to continue extrapolating the representation of the target forward in the current direction of motion). As long as displacement was generally useful for motor responding, it could have been selected for even if there were occasional cases in which displacement did not accurately anticipate a specific target.

The notion of a representational reflex arc that bridges perception and action is consistent with the developing literature on "forward models." Such models code responses in a way that reflects the anticipated sensory consequences of those responses and involve inputs from afferent and efferent sources (Desmurget \& Grafton, 2003). A combining of afferent and efferent information in forward models is consistent with motor theories of perception (e.g., Liberman \& Mattingly, 1985), models of perception and action (e.g., Brass, Bekkering, Wohlschläger, \& Prinz, 2000), ideomotor principles of human action (e.g., Hommel, 1996), and notions regarding the anticipatory nature of consciousness and the dynamics of sensorimotor control systems (e.g., Jordan, 1998). Just as such theories and models suggest that perception of environmental events can activate action representations or that environmental events initially produced by certain actions subsequently develop the capacity of activating those actions, so too might the experience of physical principles and other expectations regarding event structure activate displacement in the representation of a target. Although some discussions of representational momentum can be read as implying that displacement involves the separation of afferent and efferent systems (i.e., displacement occurs between perception and action), representational momentum and the representational reflex arc notion are compatible with the possibility that displacement reflects an integrated or common system of representation (i.e., displacement occurs in the overlap of perception and action).

The magnitude of displacement is usually smaller than what might be expected if the actual effects of physical principles on a physical target were extrapolated, and this has been used as a criticism of theories of displacement that postulate an extrapolation of the effects of physical principles (see, e.g., Ranney, 1989). However, given that a target may not always behave in the expected manner (e.g., a prey animal may change its direction of flight), it could be argued that the most adaptive displacement would be one that biased the observer in the expected direction but that would not significantly delay responding if the target behaved in an unexpected manner. A displacement smaller than the actual influence of the physical principle on an analogous physical object could provide an ideal compromise level of displacement: A smaller displacement could facilitate responding when a target behaved as expected but would offer less interference with responding when a target did not behave as expected. Therefore, a relatively smaller displacement would not reflect a lack of extrapolation or a lack of incorporation of the effects of physical principles but would actually reflect a more optimal level of such extrapolation or incorporation (cf. Finke \& Freyd, 1989). However, and as was noted earlier, displacement reflects a wide range of influences and information in addition to the effects of implied physical principles, and so it should not be surprising that the magnitude of displacement is not the same as the magnitude of the effects of physical principles on a physical target.

The notion that displacement bridges the gap between perception and action suggests that displacement might be especially robust when responses to a target involve motor actions such as reaching or grasping. As was noted earlier, Kerzel and Gegenfurtner (2003) had observers indicate the final location of a horizontally moving target by reaching and touching the display or by judging a subsequently presented probe, and reaching resulted in larger forward displacement than did probe judgment (see also Ashida, 2004; Kerzel, 2003c). On the basis of the larger displacement they observed with motor responses than with nonmotor responses, Kerzel and Gegenfurtner suggested that neuronal latencies are not compensated for in the early stages of visual processing but are compensated for at a relatively late stage of processing when retinotopic information is transformed into egocentric information that is used for motor responses. The idea that displacement occurs when retinotopic information is transformed into egocentric information is consistent with the notion that displacement allows more effective motor responses to be generated but does not appear to be consistent with the notion that compensation for neural delays occurs in the early stages of visual processing (e.g., in the flash-lag effect; Nijhawan, 2002). Even so, the notion that displacement bridges the gap between perception and action is consistent with a compensation for neural delays, regard- 
less of the level of processing at which such compensation occurs.

Although displacement of the representation might help bridge perception and action, once a response has reached the target (or once it becomes clear that no response from the observer will be issued), there is no need for the representation to stay permanently displaced. Indeed, a longer lasting displacement could be maladaptive if it led to a permanent distortion of the information regarding the target that was subsequently encoded into long-term memory. What might be most useful is a displacement that existed only during that brief time in which an immediate response would be made and then decreased before that distorted information could be encoded into a more longterm storage. The findings of Freyd and Johnson (1987) and, possibly, of Jordan et al. (2002) that the magnitude of representational momentum declines after an early or initial peak are consistent with this idea; an early or initial peak of representational momentum followed by a decrease in the magnitude of forward displacement would preserve the fidelity of a more long-term memory (and also predicts that displacement shown in Kerzel, 2000, and Halpern \& Kelly, 1993, would have declined with longer retention intervals). Analogously, once the work of the reflex arc has been completed and the observer has responded appropriately to the stimulus, reflexive motor commands are no longer issued. After the initial action, both physiological reflexive activity and displacement in mental representation return to a zero level.

\section{Displacement Within the Larger Computational Theory of Perception}

If perception is considered at the level of computational theory, one purpose of perception would presumably be to provide the observer with information regarding the environment (and specific stimuli in the environment) that is necessary for survival. Any strategy or bias that could increase the accuracy of spatial localization of stimuli in the environment could potentially facilitate interaction with (or avoidance of) specific stimuli and thus aid in survival; given that displacement temporarily adjusts the representation to reflect where a target would be when an immediate response from the observer would reach that target, displacement aids in spatial localization and so would be an adaptive strategy that could aid in survival. Furthermore, a consideration of displacement addresses Marr's (1982) criteria for a computational theory in that such a consideration specifies (1) what is computed and why (e.g., target location in the immediate future, to bridge the gap between perception and action) and (2) constraints that must be satisfied (e.g., observers' expectancies regarding the target). Because displacement bridges the gap between perception and action to ensure an increased accuracy in spatial localization and a more adaptive responding to stimuli within the environment, a consideration of displacement constitutes a seminal component of any larger computational theory of perception.

Even though different examples of displacement might arise from different types of stimuli or from activity in different cortical structures or mechanisms, those different examples of displacement might, nonetheless, reflect the same goal or purpose at the level of computational theory. For example, in an open and well-lit field, a moving target would be continuously visible, whereas in a dense forest setting in which there are numerous shadows and occluding objects, a moving target would be visible only intermittently. Motion of an object in the former case would be similar to continuous motion stimuli, and motion of an object in the latter case would be similar to implied motion stimuli. In both types of environments and with both types of stimuli, there is a common problem of the localization of stimuli, and in both types of environments and with both types of stimuli, a forward displacement in the representation could aid in the localization of stimuli. Even though processing of continuous motion and processing of implied motion might be implemented in different cortical structures or mechanisms, forward displacement resulting from either type of motion provides the same benefit of aiding localization. Similarly, displacement of targets in frozen-action photographs or downward displacement of horizontally moving targets or of stationary targets might be implemented in different cortical structures or mechanisms, but each would provide the same benefit of aiding localization. ${ }^{7}$

\section{Toward an Ultimate Theory of Displacement}

Marr (1982) suggested that a complete understanding of some process involved explanation at the levels of computational theory, representation and algorithm, and implementation; accordingly, a complete understanding of displacement will presumably involve an integration of information from a variety of influences and from each of these levels (e.g., see the discussion of motion correspondence in Dawson, 1998). A consideration of displacement at the level of computational theory provides a broad framework within which theories regarding the representation and algorithm and the implementation of displacement can be developed. However, research and theorizing regarding displacement has often proceeded in the absence of an explicit computational theory. As a result, the common problem of spatial localization of stimuli that is addressed by various instances of displacement has often not been acknowledged, and different examples of displacement (e.g., that resulting from implied motion or from continuous motion) have been suggested to reflect different phenomena. This has led some researchers to make very broad claims that seemingly apply to displacement in general but are, instead, applicable to only a specific implementation of displacement. Although a consideration of various theories of representational momentum and of displacement is beyond the scope of this review (for such a consideration, see Hubbard, in press-a), the issue of whether a general theory of displacement is possible will be briefly addressed.

Kerzel (2003b) has concluded that a single unified theory of displacement that is based on oculomotor behavior is not possible at this time. However, attempting to base a unified theory of displacement on a specific form of 
implementation (e.g., oculomotor behavior) completely misses the larger point: A cognitive theory of displacement would address how different mechanisms were employed in solving the more general goal of displacement, regardless of the specific details of representation, algorithm, or implementation. Kerzel (2003b) went on to imply that a unified theory is, therefore, not possible at all, but such a conclusion is unwarranted at this time. Even if different mechanisms account for displacement with implied motion and for displacement with continuous motion, displacement with implied motion and displacement with continuous motion need not be considered as separate phenomena. As was noted earlier, similarity of the hypothesized purpose of these displacements, as well as similarity of the effects of the same variables (e.g., velocity), suggests that displacement with implied motion and displacement with continuous motion are different manifestations of a more general phenomenon. It might be more useful to consider what more general mechanism could give rise to the same pattern of distortion despite potential differences in the implementation (e.g., in different cortical structures) of that distortion in different domains or for different types of targets.

As was noted earlier, and given that high-level variables influence displacement, it seems reasonable to speculate that whatever theory of displacement is ultimately developed will need to be a relatively high-level theory or contain high-level components. If a high-level mechanism biases mental representation in ways consistent with previous experience, that mechanism could produce displacement in memory for the final location of a target by accessing information from a variety of high-level (e.g., expectations and beliefs regarding target motion) and lowlevel (e.g., pursuit eye movements) sources and might also exhibit control of other high-level (e.g., allocation of attention) and low-level (e.g., anticipatory eye movements) processes. This information would automatically adjust mental representation to reflect what might be perceived in the next instant of time or with the next ocular fixation, and the result of this adjustment would be a displacement that optimized an observer's ability to localize and appropriately respond to stimuli. There is ample precedent within the psychological and cognitive literatures for postulating such a high-level mechanism that biases mental representation; numerous theorists have postulated that other biases and distortions in encoding, storage, and retrieval reflect operations of high-level schemata, scripts, and frames.

\section{PART V Summary and Conclusions}

Parts I and II provided overviews of displacement in memory for the final position of a target and of the variables known to influence such displacement. Although the initial investigations of displacement yielded data consistent with the hypothesis that displacement reflects an internalization or incorporation of the physical principles that would act on a physical object, subsequent investiga- tions revealed that displacement is often influenced by variables unrelated to the physical principles that would act on a physical object. Instead, displacement reflects a wide range of influences and information based on characteristics of the target (e.g., velocity, direction of motion, identity, shape, or mass/size), display (e.g., surface form of the target, retention interval, or response measure), context (e.g., the presence of a larger surrounding context, schematic information relevant to that context, the presence of a landmark or briefly presented distractor, expectations regarding future motion of target, or attributions of the source of target motion), and observer (e.g., the allocation of attention, whether or not a visual stimulus is visually tracked, activation of action plans, age of the observer, or the presence of pathology). This new taxonomy involving characteristics of the target, display, context, and observer explicitly suggests that displacement is multiply determined and underscores that information related to physical principles is just one of many potential influences on displacement.

The review of the literature in Parts I and II supported a number of broad-based conclusions regarding displacement that were discussed in Part III. As was just noted, displacement of a given target does not usually reflect a single cause but usually reflects a wide range of influences, and displacement for different targets can result from different combinations of different influences. Displacement does not accord with predictions based solely on an internalization or incorporation of objective physical principles, although information consistent with the subjective experience of physical principles can be one of several variables that contribute to displacement. In general, displacement of a target appears to reflect an observer's understanding of the larger event structure within which that target is embedded. Displacement is exhibited in memory for visual or auditory stimuli, evoked by a variety of stimulus formats, and can be measured with a variety of visual or motor responses. Although numerous low-level systems for processing different stimulus formats, dimensions, and modalities might each contain a specific or unique mechanism for displacement, it is more parsimonious to consider displacement as reflecting high-level processes that are influenced by an observer's expectations, beliefs, and knowledge. Even so, an observer's explicit knowledge of physical principles does not appear to be related to displacement, and so some aspects of displacement may reflect relatively modular or isolated processes.

Part IV suggested that displacement aids in the spatial localization of stimuli in the environment by helping to bridge the gap between perception and action. More specifically, displacement adjusts the representation of a target to reflect where that target would (most likely) be at the moment an immediate motor response from the observer would reach the target. Even though different examples of displacement might result from different types of stimuli or from different mechanisms, consideration of a possible computational theory of displacement suggests that each of those different examples of displacement re- 
flects a more general process related to aiding spatial localization. Given that accurate spatial localization is necessary for adaptive interactions with the environment, a consideration of displacement should ultimately provide a key component of theories of the perception and representation of the world. The usefulness or appropriateness of any such cognitive theory of displacement will not require a given outcome (e.g., forward displacement) to be instantiated by the same mechanism each time that outcome occurs but should address how different mechanisms were employed in solving the more general goal of displacement (regardless of the specific details of representation, algorithm, or implementation). Although ample data on displacement have been collected, much theoretical and conceptual work remains to be done.

\section{REFERENCES}

Actis-Grosso, R., \& Stucchi, N. (2003). Shifting the start: Backward mislocation of the initial position of a motion. Journal of Experimental Psychology: Human Perception \& Performance, 29, 675-691.

Actis-Grosso, R., Stucchi, N., \& Vicario, G. B. (1996). On the length of trajectories for moving dots. In S. C. Masin (Ed.), Fechner Day 1996: Proceedings of the 12th Annual Meeting of the International Society for Psychophysics (pp. 185-190). Padua, Italy: International Society for Psychophysics.

Amorim, M. A., Lang, W., Lindinger, G., Mayer, D., Deecke, L., \& Berthoz, A. (2000). Modulation of spatial orientation by mental imagery instructions: A MEG study of representational momentum. Journal of Cognitive Neuroscience, 12, 569-582.

AsHidA, H. (2004). Action-specific extrapolation of target motion in human visual system. Neuropsychologia, 42, 1515-1524.

Berry, M. J., Jr., Brivanlou, I. H., Jordan, T. A., \& Meister, M. (1999). Anticipation of moving stimuli by the retina. Nature, $\mathbf{3 9 8}$, 334-338.

Bertamini, M. (1993). Memory for position and dynamic representations. Memory \& Cognition, 21, 449-457.

Brass, M., Bekkering, H., Wohlschläger, A., \& Prinz, W. (2000). Compatibility between observed and executed finger movements: Comparing symbolic, spatial, and imitative cues. Brain \& Cognition, 44, 124-143.

Brehaut, J. C., \& Tipper, S. P. (1996). Representational momentum and memory for luminance. Journal of Experimental Psychology: Human Perception \& Performance, 22, 480-501.

Brouwer, A. M., Franz, V. H., \& Thornton, I. M. (2004). Representational momentum in perception and grasping: Translating versus transforming objects. Journal of Vision, 4, 575-584.

Bryant, D. J., \& Subbiah, I. (1994). Subjective landmarks in perception and memory for spatial location. Canadian Journal of Experimental Psychology, 48, 119-139.

Conners, F. A., Wyatt, B. S., \& Dulaney, C. L. (1998). Cognitive representation of motion in individuals with mental retardation. American Journal on Mental Retardation, 102, 438-450.

Cooper, L. A., \& Munger, M. P. (1993). Extrapolations and remembering positions along cognitive trajectories: Uses and limitations of analogies to physical momentum. In N. Eilan, R. McCarthy, \& B. Brewer (Eds.), Spatial representation: Problems in philosophy and psychology (pp. 112-131). Cambridge, MA: Blackwell.

Dawson, M. R. W. (1998). Understanding cognitive science. Malden, MA: Blackwell.

Desmurget, M., \& Grafton, S. (2003). Feedback or feedforward control: End of a dichotomy. In S. H. Johnson-Frey (Ed.), Taking action: Cognitive neuroscience perspectives on intentional acts (pp. 289338). Cambridge, MA: MIT Press.

FAust, M. (1990). Representational momentum: A dual process perspective. Unpublished doctoral dissertation, University of Oregon, Eugene.

FaVRetto, A. (2002). Displaced representations of targets undergo- ing luminance transformations. Unpublished doctoral dissertation, University of Trieste.

Favretto, A., Hubbard, T. L., Brandimonte, M. A., \& Gerbino, W. (1999). Effects of background luminance on representational momentum for lightness [Abstract]. Perception, 28(Suppl.), 77.

Finke, R. A., \& FREYD, J. J. (1985). Transformations of visual memory induced by implied motions of pattern elements. Journal of Experimental Psychology: Learning, Memory, \& Cognition, 11, 780-794.

FInKE, R. A., \& FREYD, J. J. (1989). Mental extrapolation and cognitive penetrability: Reply to Ranney and proposals for evaluative criteria. Journal of Experimental Psychology: General, 118, 403-408.

Finke, R. A., Freyd, J. J., \& ShyI, G. C. W. (1986). Implied velocity and acceleration induce transformations of visual memory. Journal of Experimental Psychology: General, 115, 175-188.

FINKE, R. A., \& SHYI, G. C. W. (1988). Mental extrapolation and representational momentum for complex implied motions. Journal of Experimental Psychology: Learning, Memory, \& Cognition, 14, 112-120.

FODOR, J. A. (1983). The modularity of mind. Cambridge, MA: MIT Press.

Foster, D. H., \& GRAVANO, S. (1982). Overshoot of curvature in visual apparent motion. Perception \& Psychophysics, 31, 411-420.

FreYD, J. J. (1983). The mental representation of movement when static stimuli are viewed. Perception \& Psychophysics, 33, 575-581.

FreYd, J. J. (1987). Dynamic mental representations. Psychological Review, 94, 427-438.

Freyd, J. J., \& Finke, R. A. (1984). Representational momentum. Journal of Experimental Psychology: Learning, Memory, \& Cognition, 10, 126-132.

FreYd, J. J., \& FinKE, R. A. (1985). A velocity effect for representational momentum. Bulletin of the Psychonomic Society, 23, 443-446.

Freyd, J. J., \& Johnson, J. Q. (1987). Probing the time course of representational momentum. Journal of Experimental Psychology: Learning, Memory, \& Cognition, 13, 259-269.

Freyd, J. J., \& Jones, K. T. (1994). Representational momentum for a spiral path. Journal of Experimental Psychology: Learning, Memory, \& Cognition, 20, 968-976.

Freyd, J. J., Kelly, M. H., \& DeKay, M. L. (1990). Representational momentum in memory for pitch. Journal of Experimental Psychology: Learning, Memory, \& Cognition, 16, 1107-1117.

Freyd, J. J., \& Miller, G. F. (1992, November). Creature motion. Paper presented at the 33rd Annual Meeting of the Psychonomic Society, St. Louis.

Freyd, J. J., \& Pantzer, T. M. (1995). Static patterns moving in the mind. In S. M. Smith, T. B. Ward, \& R. A. Finke (Eds.), The creative cognition approach (pp. 181-204). Cambridge, MA: MIT Press.

Freyd, J. J., PAntzer, T. M., \& Cheng, J. L. (1988). Representing statics as forces in equilibrium. Journal of Experimental Psychology: General, 117, 395-407.

Futterweit, L. R., \& Beilin, H. (1994). Recognition memory for movement in photographs: A developmental study. Journal of Experimental Child Psychology, 57, 163-179.

Gelman, R., Durgin, F., \& Kaufman, L. (1995). Distinguishing between animates and inanimates: Not by motion alone. In D. Sperber, D. Premack, \& A. J. Premack (Eds.), Causal cognition: A multidisciplinary debate (pp. 150-184). Oxford: Oxford University Press, Clarendon Press.

Getzmann, S. (2005). Representational momentum in spatial hearing does not depend on eye movements. Experimental Brain Research, 165, 229-238.

Getzmann, S., Lewald, J., \& Guski, R. (2004). Representational momentum in spatial hearing. Perception, 33, 591-599.

Gottesman, C. V., \& Intraub, H. (2002). Surface construal and the mental representation of scenes. Journal of Experimental Psychology: Human Perception \& Performance, 28, 589-599.

Gray, R., \& Thornton, I. M. (2001). Exploring the link between time to collision and representational momentum. Perception, 30, 1007 1022.

Halpern, A. R., \& Kelly, M. H. (1993). Memory biases in left versus right implied motion. Journal of Experimental Psychology: Learning, Memory, \& Cognition, 19, 471-484.

Hayes, A. E., \& Freyd, J. J. (2002). Representational momentum when attention is divided. Visual Cognition, 9, 8-27. 
Hayes, A. E., Sacher, G., Thornton, I. M., Sereno, M. E., \& Freyd, J. J. (1996). Representational momentum in depth using stereopsis [ARVO Abstract 2120]. Investigative Ophthalmology \& Visual Science, 37(Suppl. 3), S467.

Heider, F., \& Simmel, M. (1944). An experimental study of apparent behavior. American Journal of Psychology, 57, 243-259.

Hommel, B. (1996). Toward an action-concept model of stimulusresponse compatibility. In B. Hommel \& W. Prinz (Eds.), Theoretical issues in stimulus-response compatibility (pp. 281-320). Amsterdam: North-Holland.

Hubbard, T. L. (1990). Cognitive representation of linear motion: Possible direction and gravity effects in judged displacement. Memory \& Cognition, 18, 299-309.

Hubbard, T. L. (1993a). Auditory representational momentum: Musical schemata and modularity. Bulletin of the Psychonomic Society, 31, 201-204.

HubBard, T. L. (1993b). The effects of context on visual representational momentum. Memory \& Cognition, 21, 103-114.

HubBard, T. L. (1994). Judged displacement: A modular process? American Journal of Psychology, 107, 359-373.

HubBard, T. L. (1995a). Auditory representational momentum: Surface form, velocity, and direction effects. American Journal of Psychology, 108, 255-274.

HuBbard, T. L. (1995b). Cognitive representation of motion: Evidence for representational friction and gravity analogues. Journal of Experimental Psychology: Learning, Memory, \& Cognition, 21, 241-254.

HuBbard, T. L. (1995c). Environmental invariants in the representation of motion: Implied dynamics and representational momentum, gravity, friction, and centripetal force. Psychonomic Bulletin \& Review, 2, 322-338

Hubbard, T. L. (1996a). Displacement in depth: Representational momentum and boundary extension. Psychological Research, 59, 33-47.

HubBard, T. L. (1996b). Representational momentum, centripetal force, and curvilinear impetus. Journal of Experimental Psychology: Learning, Memory, \& Cognition, 22, 1049-1060.

Hubbard, T. L. (1997). Target size and displacement along the axis of implied gravitational attraction: Effects of implied weight and evidence of representational gravity. Journal of Experimental Psychology: Learning, Memory, \& Cognition, 23, 1484-1493.

HubBard, T. L. (1998a). Representational momentum and other displacements in memory as evidence for nonconscious knowledge of physical principles. In S. R. Hameroff, A. W. Kaszniak, \& A. C. Scott (Eds.), Toward a science of consciousness II: The second Tucson discussions and debates (pp. 505-512). Cambridge, MA: MIT Press.

HuBBaRD, T. L. (1998b). Some effects of representational friction, target size, and memory averaging on memory for vertically moving targets. Canadian Journal of Experimental Psychology, 52, 44-49.

HuBBaRD, T. L. (1999). How consequences of physical principles influence mental representation: The environmental invariants hypothesis. In P. R. Killeen \& W. R. Uttal (Eds.), Fechner Day 99: The end of 20th century psychophysics. Proceedings of the 15thAnnual Meeting of the International Society for Psychophysics (pp. 274-279). Tempe, AZ: International Society for Psychophysics.

Hubbard, T. L. (2001). The effect of height in the picture plane on the forward displacement of ascending and descending targets. Canadian Journal of Experimental Psychology, 55, 325-330.

HubBard, T. L. (2004). The perception of causality: Insights from Michotte's launching effect, naive impetus theory, and representational momentum. In A. M. Oliveira, M. P. Teixeira, G. F. Borges, \& M. J. Ferro (Eds.), Fechner Day 2004 (pp. 116-121). Coimbra, Portugal: International Society for Psychophysics.

Hubbard, T. L. (in press-a). Approaches to representational momentum: Theories and models. In R. Nijhawan \& B. Khurana (Eds.), Space and time perception and action. Cambridge: Cambridge University Press.

HubBard, T. L. (in press-b). Bridging the gap: Possible roles and contributions of representational momentum. Psicológica.

Hubbard, T. L., \& Bharucha, J. J. (1988). Judged displacement in apparent vertical and horizontal motion. Perception \& Psychophysics, 44, 211-221.
Hubbard, T. L., \& Blessum, J. A. (2001). A structural dynamic of form: Displacements in memory for the size of an angle. Visual Cognition, 8, 725-749.

Hubbard, T. L., Blessum, J. A., \& Ruppel, S. E. (2001). Representational momentum and Michotte's "launching effect" paradigm. Journal of Experimental Psychology: Learning, Memory, \& Cognition, 27, 294-301.

Hubbard, T. L., \& Courtney, J. R. (in press). Evidence suggestive of separate visual dynamics in perception and in memory. In L. Albertazzi (Ed.), Visual thought: The depictive space of the mind. Amsterdam: Benjamins.

Hubbard, T. L., \& Favretto, A. (2003). Naive impetus and Michotte's "tool effect": Evidence from representational momentum. Psychological Research, 67, 134-152.

Hubbard, T. L., Matzenbacher, D. L., \& Davis, S. E. (1999). Representational momentum in children: Dynamic information and analogue representation. Perceptual \& Motor Skills, 88, 910-916.

Hubbard, T. L., \& Motes, M. A. (2002). Does representational momentum reflect a distortion of the length or the endpoint of a trajectory? Cognition, 82, B89-B99.

Hubbard, T. L., \& Motes, M. A. (2005). An effect of context on whether memory for initial position exhibits a Fröhlich effect or an onset repulsion effect. Quarterly Journal of Experimental Psychology, 58A, 961-979.

Hubbard, T. L., \& Ruppel, S. E. (1999). Representational momentum and landmark attraction effects. Canadian Journal of Experimental Psychology, 53, 242-256.

Hubbard, T. L., \& RuPPEL, S. E. (2000). Spatial memory averaging, the landmark attraction effect, and representational gravity. Psychological Research, 59, 41-55.

Hubbard, T. L., \& Ruppel, S. E. (2002). A possible role of naïve impetus in Michotte's "launching effect": Evidence from representational momentum. Visual Cognition, 9, 153-176.

Hubbard, T. L., Ruppel, S. E., \& Courtney, J. R. (2005). The force of appearance: Gamma movement, naive impetus, and representational momentum. Psicológica, 26, 209-228.

INTRAUB, H. (2002). Anticipatory spatial representation of natural scenes: Momentum without movement? Visual Cognition, 9, 93-119.

Intraub, H., Bender, R. S., \& MANGels, J. A. (1992). Looking at pictures but remembering scenes. Journal of Experimental Psychology: Learning, Memory, \& Cognition, 18, 180-191.

Intraub, H., Gottesman, C. V., \& Bills, A. J. (1998). Effects of perceiving and imaging scenes on memory for pictures. Journal of Experimental Psychology: Learning, Memory, \& Cognition, 24, 186-201.

Jarrett, C. B., Phillips, M., Parker, A., \& Senior, C. (2002). Implicit motion perception in schizotypy and schizophrenia: A representational momentum study. Cognitive Neuropsychiatry, 7, 1-14.

Johansson, G. (1973). Visual perception of biological motion and a model for its analysis. Perception \& Psychophysics, 14, 201-211.

Johnston, H. M., \& Jones, M. R. (in press). Higher-order pattern structure influences auditory representational momentum. Journal of Experimental Psychology: Human Perception \& Performance.

JONES, L. A. (1988). Motor illusions: What do they reveal about proprioception? Psychological Bulletin, 103, 72-86.

Joordens, S., SpaleK, T. M., Razmy, S., \& van Duijn, M. (2004). A Clockwork Orange: Compensation opposing momentum in memory for location. Memory \& Cognition, 32, 39-50.

JoRDAN, J. S. (1998). Recasting Dewey's critique of the reflex-arc concept via a theory of anticipatory consciousness: Implications for theories of perception. New Ideas in Psychology, 16, 165-187.

Jordan, J. S., \& KNOBLICH, G. (2004). Spatial perception and control. Psychonomic Bulletin \& Review, 11, 54-59.

Jordan, J. S., Stork, S., Knuf, L., Kerzel, D., \& Müsseler, J. (2002). Action planning affects spatial localization. In W. Prinz \& B. Hommel (Eds.), Common mechanisms in perception and action: Attention and performance XIX (pp. 158-176). New York: Oxford University Press.

Kelly, M. H., \& Freyd, J. J. (1987). Explorations of representational momentum. Cognitive Psychology, 19, 369-401.

Kerzel, D. (2000). Eye movements and visible persistence explain the 
mislocalization of the final position of a moving target. Vision Research, 40, 3703-3715.

Kerzel, D. (2002a). Attention shifts and memory averaging. Quarterly Journal of Experimental Psychology, 55A, 425-443.

Kerzel, D. (2002b). The locus of "memory displacement" is at least partially perceptual: Effects of velocity, expectation, friction, memory averaging, and weight. Perception \& Psychophysics, 64, 680-692.

Kerzel, D. (2002c). A matter of design: No representational momentum without predictability. Visual Cognition, 9, 66-80.

KerzeL, D. (2003a). Attention maintains mental extrapolation of target position: Irrelevant distractors eliminate forward displacement after implied motion. Cognition, 88, 109-131.

KERZEL, D. (2003b). Mental extrapolation of target position is strongest with weak motion signals and motor responses. Vision Research, 43, 2623-2635.

Kerzel, D. (2003c). Centripetal force draws the eyes, not memory of the target, toward the center. Journal of Experimental Psychology: Learning, Memory, \& Cognition, 29, 458-466.

Kerzel, D., \& Gegenfurtner, K. R. (2003). Neuronal processing delays are compensated in the sensorimotor branch of the visual system. Current Biology, 13, 1975-1978.

Kerzel, D., Jordan, J. S., \& Müsseler, J. (2001). The role of perception in the mislocalization of the final position of a moving target. Journal of Experimental Psychology: Human Perception \& Performance, 27, 829-840.

Koseleff, P. (1957). Studies in the perception of heaviness: I. Some relevant facts concerning the size-weight-effect (SWE). Acta Psychologica, 13, 242-252.

KourtZI, Z., \& KANwISHER, N. (2000). Activation in human MT/MST for static images with implied motion. Journal of Cognitive Neuroscience, 12, 1-8.

Kozhevnikov, M., \& Hegarty, M. (2001). Impetus beliefs as default heuristics: Dissociation between explicit and implicit knowledge about motion. Psychonomic Bulletin \& Review, 8, 439-453.

Liberman, A. M., \& Mattingly, I. G. (1985). The motor theory of speech perception revised. Cognition, 21, 1-36.

MARR, D. (1982). Vision. New York: Freeman.

McCloskey, M., \& Kohl, D. (1983). Naive physics: The curvilinear impetus principle and its role in interactions with moving objects. Journal of Experimental Psychology: Learning, Memory, \& Cognition, 9, 146-156.

McIntyre, J., Zago, M., Berthoz, A., \& Lacquaniti, F. (2001). Does the brain model Newton's laws? Nature Neuroscience, 4, 693-694.

Michoтte, A. (1963). The perception of causality (T. R. Miles \& E. Miles, Trans.). New York: Basic Books. (Original work published 1946)

Milner, A. D., \& Goodale, M. A. (1995). The visual brain in action. New York: Oxford University Press.

Munger, M. P., \& Minchew, J. H. (2002). Parallels between remembering and predicting an object's location. Visual Cognition, 9, 177-194.

Munger, M. P., \& Owens, T. R. (2004). Representational momentum and the flash-lag effect. Visual Cognition, 11, 81-103.

Munger, M. P., Owens, T. R., \& Conway, J. E. (2005). Are boundary extension and representational momentum related? Visual Cognition, 12, 1041-1056.

Munger, M. P., Solberg, J. L., \& Horrocks, K. K. (1999). The relationship between mental rotation and representational momentum. Journal of Experimental Psychology: Learning, Memory, \& Cognition, 25, 1557-1568.

Munger, M. P., Solberg, J. L., Horrocks, K. K., \& Preston, A. S. (1999). Representational momentum for rotations in depth: Effects of shading and axis. Journal of Experimental Psychology: Learning, Memory, \& Cognition, 25, 157-171.

Müsseler, J., Stork, S., \& Kerzel, D. (2002). Comparing mislocalizations with moving stimuli: The Fröhlich effect, the flash-lag, and representational momentum. Visual Cognition, 9, 120-138.

NAGAI, M., KAZAI, K., \& YAGI, A. (2002). Larger forward memory shift in the direction of gravity. Visual Cognition, 9, 28-40.

NAGAI, M., \& YAGI, A. (2001). The pointedness effect on representational momentum. Memory \& Cognition, 29, 91-99.
NiJHawAN, R. (2002). Neural delays, visual motion and the flash-lag effect. Trends in Cognitive Sciences, 6, 387-393.

PoljanseK, A. (2002). The effect of motion acceleration on displacement of continuous and staircase motion in the frontoparallel plane. Psiholoska Obzorja/Horizons of Psychology, 11, 7-21.

Pratt, J., SpaleK, T. M., \& Bradshaw, F. (1999). The time to detect targets at inhibited and noninhibited locations: Preliminary evidence for attentional momentum. Journal of Experimental Psychology: Human Perception \& Performance, 25, 730-746.

Premack, D., \& Premack, A. J. (1995). Intention as psychological cause. In D. Sperber, D. Premack, \& A. J. Premack (Eds.), Causal cognition: A multidisciplinary debate (pp. 185-199). Oxford: Oxford University Press, Clarendon Press.

Prinz, W. (1992). Why don't we perceive our brain states? European Journal of Cognitive Psychology, 4, 1-20.

PRINZ, W. (1997). Perception and action planning. European Journal of Cognitive Psychology, 9, 129-154.

RANNEY, M. (1989). Internally represented forces may be cognitively penetrable: Comment on Freyd, Pantzer, and Cheng (1988). Journal of Experimental Psychology: General, 118, 399-402.

RaO, H., Han, S., Jiang, Y., Xue, Y., Gu, H., Cui, Y., \& GaO, D. (2004). Engagement of the prefrontal cortex in representational momentum: An fMRI study. NeuroImage, 23, 98-103.

Reed, C. L., \& Vinson, N. G. (1996). Conceptual effects on representational momentum. Journal of Experimental Psychology: Human Perception \& Performance, 22, 839-850.

Sakata, H., Kusunoki, M., \& TANAKa, Y. (1993). Neural mechanisms of perception of linear and rotary movement in depth in the parietal association cortex of the monkey. In T. Ono, L. R. Squire, M. E. Raichle, D. I. Perrett, \& M. Fukuda (Eds.), Brain mechanisms of perception and memory (pp. 166-182). New York: Oxford University Press.

Senior, C., Barnes, J., Giampietroc, V., Simmons, A., Bullmore, E. T., Brammer, M., \& David, A. S. (2000). The functional neuroanatomy of implicit-motion perception or "representational momentum." Current Biology, 10, 16-22.

Senior, C., Ward, J., \& David, A. S. (2002). Representational momentum and the brain: An investigation of the functional necessity of V5/MT. Visual Cognition, 9, 81-92.

Shiffrar, M., \& FreYd, J. J. (1990). Apparent motion of the human body. Psychological Science, 1, 257-264.

Shiffrar, M., \& FreYd, J. J. (1993). Timing and apparent motion path choice with human body photographs. Psychological Science, 4, 379384.

Sillito, A. M., Jones, H. E., Gerstein, G. L., \& West, D. C. (1994). Feature-linked synchronization of thalamic relay cell firing induced by feedback from the visual cortex. Nature, 369, 479-482.

Snyder, J. J., Schmidt, W. C., \& Kingstone, A. (2001). Attentional momentum does not underlie the inhibition of return. Journal of Experimental Psychology: Human Perception \& Performance, 27, 1420-1432.

Stewart, J. A. (1982). Perception of animacy. Unpublished doctoral dissertation, University of Pennsylvania.

Thornton, I. M. (1998). The perception of dynamic human faces. Unpublished doctoral dissertation. University of Oregon, Eugene.

Thornton, I. M. (2002). The onset repulsion effect. Spatial Vision, 15, 219-243.

Thornton, I. M., \& Hayes, A. E. (2004). Anticipating action in complex scenes. Visual Cognition, 11, 341-370.

Thornton, I. M., \& Hubbard, T. L. (Eds.) (2002). Representational momentum: New findings, new directions. Hove, U.K.: Taylor \& Francis, Psychology Press.

Verfaillie, K., \& Daems, A. (2002). Representing and anticipating human actions in vision. Visual Cognition, 9, 217-232.

Verfaillie, K., De Troy, A., \& Van Rensbergen, J. (1994). Transsaccadic integration of biological motion. Journal of Experimental Psychology: Learning, Memory, \& Cognition, 20, 649-670.

Verfaillie, K., \& D'Ydewalle, G. (1991). Representational momentum and event course anticipation in the perception of implied periodical motions. Journal of Experimental Psychology: Learning, Memory, \& Cognition, 17, 302-313. 
Vinson, N. G., \& ReED, C. L. (2002). Sources of object-specific effects in representational momentum. Visual Cognition, 9, 41-65.

White, H., Minor, S. W., Merrell, J., \& Smith, T. (1993). Representational-momentum effects in the cerebral hemispheres. Brain \& Cognition, 22, 161-170.

Whitney, D., \& Cavanagh, P. (2002). Surrounding motion affects the perceived locations of moving stimuli. Visual Cognition, 9, 139-152.

\section{NOTES}

1. Studies of biological motion (e.g., Johansson, 1973) and of apparent motion using biological stimuli (e.g., Shiffrar \& Freyd, 1990, 1993), suggest that the motion processing of animate targets differs from the motion processing of inanimate targets. Also, observers may attribute intentionality to targets that move in a more animate fashion (e.g., Heider \& Simmel, 1944; Premack \& Premack, 1995), and an observer's beliefs regarding the intentions of targets perceived or believed to be animate could provide additional context capable of influencing displacement of such targets (e.g., animate targets may be perceived as less likely to follow purely mechanical paths; Stewart, 1982; but see Gelman, Durgin, \& Kaufman, 1995)

2. Motion depicted in a computer-generated animation is never actually smooth motion or continuous motion, because computer-generated motion involves discrete presentations of a static target at one location and then a redrawing of that static target at a nearby location. However, if the separate presentations occur quickly enough, a smooth and continuous apparent motion is perceived. When researchers on displacement speak of smooth motion or of continuous motion, they are generally referring to a case in which the differences between successive presentations of a static target located at a slightly different position on each presentation are not perceivable, and so the target appears to exhibit smooth and continuous motion.

3. It is important to distinguish between two senses of extrapolation that are used in the displacement literature. One sense involves a conscious explicit prediction of a future location, and that is the sense used in Finke and Shyi (1988). A second, and more common, sense involves an unconscious and automatic adjustment of the representation (e.g., as in Hayes \& Freyd, 2002).

4. In Hubbard (2001), targets ascended or descended at a constant velocity, but displacement patterns reflected how a rising physical object would decelerate and a falling physical object accelerate. In Hubbard (1995b, 1998b), targets moved at a constant velocity before and after encountering friction, but displacement patterns reflected how a physical object encountering friction would decelerate afterward. However, displacement is not merely an artifact of inaccurate displays, since displacement is also found when computer animations correctly reflect physical principles (e.g., as in Kozhevnikov \& Hegarty, 2001).

5. In addition to the empirical data suggesting that eye movements do not cause displacement, there are also a number of methodological arguments against an eye movement-based theory of representational momentum. Studies supporting the notion that displacement may be attributable to eye movements have often included a condition in which observers fixated a point some distance away from the target. However, it would not be surprising that eye movements provide input that will be used by memory or by some other high-level cognitive system responsible for displacement; if that input is disrupted, displacement will also be disrupted. Such disruption, though, does not provide sufficient evidence to conclude that eye movements are a necessary or a sufficient cause for displacement (e.g., just as disruption of a given cognitive process by a lesion to a specific brain structure does not provide sufficient evidence to conclude that that specific brain structure is where that cognitive process was localized). Also, having a centrally positioned fixation point remain present throughout an entire experimental trial could provide a landmark, and given that displacement may be influenced by the direction of target motion relative to a landmark, the visible presence of a fixation point could further bias judgment of remembered position. Finally, when observers view moving objects in the natural environment, those observers visually track the objects, and so forcing observers to fixate on an arbitrary point some distance away from a moving target whose location they know they will be asked to judge further limits ecological validity.

6. It might be argued that a physiological reflex action is not "anticipatory" because reflexes are reactive: In a reflex, a stimulus impinges on the system, and via hardwired connections, a response is elicited. However, once engaged, a reflexive response may in some cases "anticipate" a subsequent cortically mediated response from the observer (e.g., a reflex "anticipates" cortical commands to remove the hand from a hot stove). In the same way, displacement may be engaged when a stimulus impinges on the system (e.g., a moving object is detected), and displacement "anticipates" a subsequent motor response to the object and adjusts the representation accordingly.

7. A related type of bias in spatial localization that might also aid spatial localization is boundary extension, in which an observer's memory for a scene is likely to include details and information that were not actually present within the boundaries of the scene but that might have been present just beyond the boundaries (e.g., Gottesman \& Intraub, 2002; Intraub, Bender, \& Mangels, 1992; Intraub, Gottesman, \& Bills, 1998). Both boundary extension and representational momentum appear to serve a similar purpose (i.e., anticipating what may be seen in the next moment or fixation), and this suggests that they may be related at the level of computational theory. Both boundary extension and representational momentum may reflect the operation of a more general mechanism that biases representation in ways consistent with past experience; like representational momentum, boundary extension may help bridge the gap between perception and action by allowing an observer to anticipate what will (probably) be seen in the immediate future (Hubbard, 1995c, 1996a, in press-b; cf. Intraub, 2002).

(Manuscript received August 11, 2004; revision accepted for publication April 22, 2005.) 